\title{
ARTICLE
}

Received 26 Dec 2015 | Accepted 26 Jul 2016 | Published 6 Oct 2016

DOI: $10.1038 /$ ncomms12713

OPEN

\section{A distinct holoenzyme organization for two-subunit pyruvate carboxylase}

Philip H. Choi ${ }^{1}$, Jeanyoung Jo ${ }^{1}$, Yu-Cheng Lin ${ }^{1}$, Min-Han Lin ${ }^{2}$, Chi-Yuan Chou ${ }^{2}$, Lars E.P. Dietrich ${ }^{1}$ \& Liang Tong ${ }^{1}$

Pyruvate carboxylase (PC) has important roles in metabolism and is crucial for virulence for some pathogenic bacteria. PC contains biotin carboxylase (BC), carboxyltransferase (CT) and biotin carboxyl carrier protein (BCCP) components. It is a single-chain enzyme in eukaryotes and most bacteria, and functions as a $500 \mathrm{kD}$ homo-tetramer. In contrast, PC is a two-subunit enzyme in a collection of Gram-negative bacteria, with the $\alpha$ subunit containing the $B C$ and the $\beta$ subunit the CT and BCCP domains, and it is believed that the holoenzyme has $\alpha_{4} \beta_{4}$ stoichiometry. We report here the crystal structures of a two-subunit PC from Methylobacillus flagellatus. Surprisingly, our structures reveal an $\alpha_{2} \beta_{4}$ stoichiometry, and the overall architecture of the holoenzyme is strikingly different from that of the homo-tetrameric PCs. Biochemical and mutagenesis studies confirm the stoichiometry and other structural observations. Our functional studies in Pseudomonas aeruginosa show that its two-subunit PC is important for colony morphogenesis.

\footnotetext{
${ }^{1}$ Department of Biological Sciences, Columbia University, New York, New York 10027, USA. ${ }^{2}$ Department of Life Sciences and Institute of Genome Sciences, National Yang-Ming University, Taipei 112, Taiwan. Correspondence and requests for materials should be addressed to L.T. (email: Itong@columbia.edu).
} 
P yruvate carboxylase (PC) catalyses the MgATP-dependent conversion of pyruvate to oxaloacetate ${ }^{1,2}$. It has important functions in gluconeogenesis, glyceroneogenesis, lipogenesis, neurotransmitter release, as well as anaplerosis to replenish the intermediates of the tricarboxylic acid (TCA) cycle. PC deficiency in humans is linked to various clinical symptoms such as lactic acidaemia and psychomotor retardation, while PC over-expression has been observed in several types of cancers ${ }^{3,4}$. The PC of the intracellular pathogen Listeria monocytogenes (LmPC) is important for growth and virulence in the host cell ${ }^{5,6}$.

$\mathrm{PC}$ is a member of the biotin-dependent carboxylase family and contains biotin carboxylase (BC), carboxyltransferase (CT) and biotin carboxyl carrier protein (BCCP) components. In mammals and most bacteria, these domains reside in a single polypeptide chain (Fig. 1a). These single-chain PC enzymes function as $500 \mathrm{kD}$ homo-tetramers and are stimulated by acetylCoA. In mammals, insulin suppresses hepatic gluconeogenesis by reducing acetyl-CoA levels and thereby $\mathrm{PC}$ activity, suggesting an important role for PC in type 2 diabetes ${ }^{7}$. The crystal structures of several single-chain PCs have defined their overall holoenzyme architecture ${ }^{6,8,9}$. The four PC molecules are arranged in two layers, in the overall shape of a diamond (Fig. 1b). The two molecules in each layer of the structure have few interactions with each other. The holoenzyme is primarily formed through BC (Fig. 1c) and CT domain dimers between the layers, which are located at alternate corners of the diamond. The structures also reveal a new domain, named the PC tetramerization (PT) domain $^{9}$ or allosteric domain ${ }^{8}$ that is important for tetramer formation by Staphylococcus aureus and human PCs (SaPC and $\mathrm{HsPC}$ ) and for interaction with acetyl-CoA $\mathrm{Co}^{8-10}$. This domain is formed by two discontinuous segments of the protein-the linker between BC and CT and that between CT and BCCP (Fig. 1a).

On the other hand, in a diverse array of Gram-negative bacteria among the phyla Proteobacteria and Aquificae (Supplementary Fig. 1), the PC enzyme is encoded by two separate genes, with the $\mathrm{BC}$ domain residing in a $52 \mathrm{kD} \alpha$ subunit and the CT and BCCP domains in a $67 \mathrm{kD} \beta$ subunit (Fig. 1a) ${ }^{11}$. The BC, CT and BCCP domains of these two-subunit PCs share high sequence identity with those of the single-chain PCs $(\sim 50 \%$ for BC and $\sim 40 \%$ for CT and BCCP), but the sequence identity is $<10 \%$ in the regions corresponding to the PT domain (Supplementary Figs 2 and 3). It is generally accepted that these two-subunit PCs form an $\alpha_{4} \beta_{4}$ complex $^{12}$, which would be equivalent to the homo-tetrameric single-chain PCs. In contrast to the single-chain PCs, however, the two-subunit enzymes are not stimulated by acetyl-CoA ${ }^{13}$.

The two-subunit PC from Pseudomonas aeruginosa ( $\mathrm{PaPC})$ is necessary for growth on $\mathrm{C} 3$ and $\mathrm{C} 6$ compounds ${ }^{14}$. In addition, transcriptional upregulation of $\mathrm{PaPC}$ and other genes by $\mathrm{PycR}$, which is encoded immediately downstream of the PC operon in the genome, is important for maintaining $P$. aeruginosa lung infection in a rat model, and PycR has been proposed as a potential target for antimicrobial therapy ${ }^{15}$. The PC from Pseudomonas fluorescens is important for the expression of small RNAs that mediate secondary metabolism and the biosynthesis of antibiotic compounds ${ }^{16}$. The two-subunit PC from Azotobacter vinelandii is necessary for growth on minimal media with glucose or sucrose as the sole carbon source, and a deletion mutant accumulates the compound poly- $\beta$ hydroxybutyrate due to a slowdown of the TCA cycle ${ }^{17,18}$.

The crystal structure of the BC subunit of Aquifex aeolicus PC has been reported ${ }^{19}$, although currently there is no structure of the holoenzyme of a two-subunit PC. We report here the structures of the two-subunit PC from Methylobacillus flagellatus (MfPC). Surprisingly, our structures reveal an $\alpha_{2} \beta_{4}$ stoichiometry for the holoenzyme, with one BC dimer and two CT dimers, and the overall architecture of the holoenzyme is strikingly different from that of the homo-tetrameric PCs. An $\alpha$ helix at the C terminus of the $\alpha$ subunit is surrounded by two four-stranded $\beta$-sheets from two $\beta$ subunits, forming a domain that is

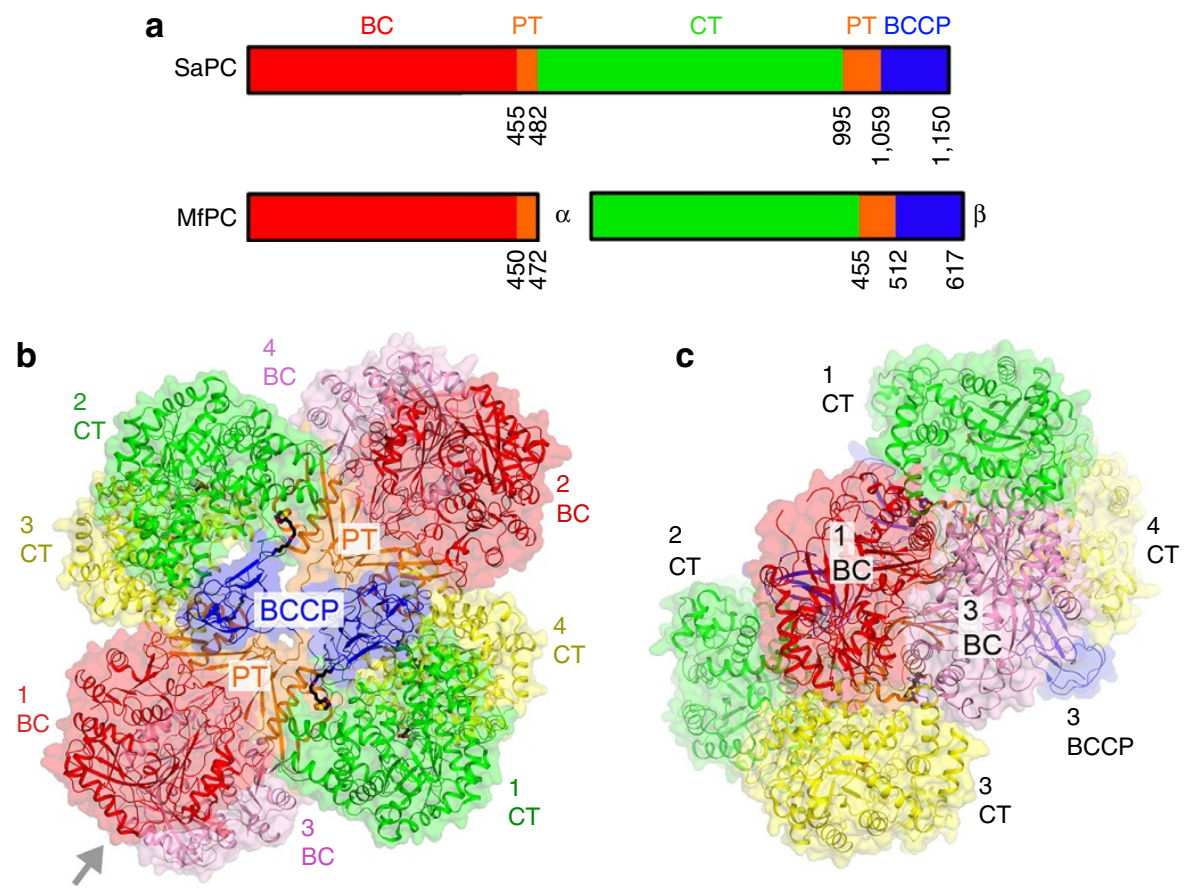

Figure 1 | Domain organization of PC. (a) Domain organization of single-chain SaPC and two-subunit MfPC. The domains are labelled and given different colours. (b) Schematic drawing of the structure of the single-chain SaPC holoenzyme ${ }^{9}$. The domains in the four monomers are coloured according to a and labelled. (c) Structure of SaPC viewed down the two-fold axis of the BC dimer, along the arrow of $\mathbf{b}$. The structure figures were produced with PyMOL (www.pymol.org). 
remarkably similar to the $\mathrm{BT}$ (BC-CT interaction) domain found in propionyl-CoA carboxylase ${ }^{20}, 3$-methylcrotonyl-CoA carboxylase $^{21}$ and acetyl-CoA carboxylase (ACC) ${ }^{22}$. Biochemical and mutagenesis studies confirm the stoichiometry, as well as other observations from the structure. Our functional studies in $P$. aeruginosa PA14 show that PC is important for colony morphogenesis and for growth on pyruvate and glucose as carbon sources.

\section{Results}

Structure determination. We screened through a collection of two-subunit PCs for their expression and crystallization behaviour, and produced crystals of MfPC ${ }^{23}$. MfPC has $65 \%$ aminoacid sequence identity with PaPC (Supplementary Figs 2 and 3). The $\alpha$ and $\beta$ subunits were over-expressed in Escherichia coli using a bicistronic plasmid, with an N-terminal hexa-histidine tag on the $\alpha$ subunit. The two subunits readily formed a stable complex, which was then purified to homogeneity. Purified MfPC was confirmed to be fully biotinylated by streptavidin gel shift assay, and had robust PC enzymatic activity (Supplementary Fig. 4). We compared the catalytic activities of MfPC and PaPC with those of the single-chain PCs LmPC and SaPC. In contrast to the single-chain PCs and consistent with previous reports, MfPC and $\mathrm{PaPC}$ are not sensitive to activation by acetyl-CoA (Supplementary Fig. 4). However, MfPC and PaPC have $\sim 10$ fold higher specific activity than LmPC and SaPC in the absence of acetyl-CoA. When acetyl-CoA is present, SaPC activity becomes comparable to those of the two-subunit PCs, while LmPC activity is still $\sim 5$-fold lower.

The MfPC crystals diffracted only to $6.6 \AA$ resolution even after extensive optimization. To improve the diffraction quality, we introduced three single-site mutations (K419A, E421A and $\mathrm{E} 422 \mathrm{~A}$ ) in the $\beta$ subunit designed to reduce the surface entropy ${ }^{24}$. These residues are located in a solvent-exposed region of the CT domain and are not strictly conserved among PC homologues (Supplementary Fig. 3). The mutations had no effect on the catalytic activity of the enzyme, but they only slightly improved the diffraction quality of the crystals.

We then removed the flexible $\mathrm{B}$ sub-domain (residues 131-201) of the BC domain and replaced it with a Gly-SerSer-Gly linker. The B domain closes down over the BC active site during catalysis but is often disordered or has high temperature factors in PC crystal structures. This deletion mutant behaved identically to the wild-type enzyme during purification, and expectedly was inactive catalytically. A mutant protein having this deletion and the three single-site changes produced a different crystal form with better diffraction, allowing us to determine the crystal structure of the MfPC holoenzyme at $3.0 \AA$ resolution (Supplementary Fig. 5) using the molecular replacement method with the SaPC structure ${ }^{9}$ as the search model. Several segments of the protein had poor electron density and were not modelled in the final structure, including the linker between the PT and BCCP domains and the linker between the CT and PT domains for one $\beta$ dimer. Only three of the four BCCP domains were observed. The crystallographic statistics are summarized in Table 1.

Using the structure of this mutant as the search model, we also determined the structure of the MfPC with only the three singlesite mutations (referred to hereafter as wild-type since the mutations did not affect the catalytic activity) at $6.6 \AA ̊$ resolution. The BC and CT domains were located individually with the molecular replacement method. After one round of refinement, there was electron density indicating the position of the PT domain, the B domain of BC and a BCCP domain in one of the $\mathrm{CT}$ active sites. Inclusion of these additional domains reduced the

\section{Table 1 | Data collection and refinement statistics.}

\begin{tabular}{|c|c|c|}
\hline & $\begin{array}{l}\text { MfPC (B domain of BC } \\
\text { deletion, K419A, E421A } \\
\text { and E422A mutation) }\end{array}$ & $\begin{array}{l}\text { MfPC (K419A, } \\
\text { E421A and } \\
\text { E422A } \\
\text { mutation) }\end{array}$ \\
\hline \multicolumn{3}{|l|}{ Data collection } \\
\hline Space group & R32 & $P 3,21$ \\
\hline \multicolumn{3}{|l|}{ Cell dimensions } \\
\hline$a, b, c(\AA)$ & $285.8,285.8,274.9$ & $\begin{array}{l}160.8,160.8 \\
227.7\end{array}$ \\
\hline$\alpha, \beta, \gamma\left(^{\circ}\right)$ & $90,90,120$ & $90,90,120$ \\
\hline Resolution $(\AA)$ & $50-3.0(3.1-3.0)^{\star}$ & $\begin{array}{l}50-6.6 \\
(6.83-6.6)\end{array}$ \\
\hline$R_{\text {merge }}$ & $8.2(60.8)$ & $8.8(>100)$ \\
\hline $\mathrm{CC}_{1 / 2}$ & $(0.67)$ & $(0.64)$ \\
\hline$l / \sigma l$ & $11.0(1.8)$ & $14.9(1.3)$ \\
\hline Completeness (\%) & $96(96)$ & $95(96)$ \\
\hline Redundancy & $2.8(2.7)$ & $6.4(6.3)$ \\
\hline \multicolumn{3}{|l|}{ Refinement } \\
\hline Resolution $(\AA)$ & $50-3.0$ & $50-6.6$ \\
\hline No. reflections & 77,247 & 6,031 \\
\hline \multicolumn{3}{|l|}{ No. of atoms } \\
\hline Protein & 21,658 & 11,277 \\
\hline Ligand/ion & 30 & 0 \\
\hline Water & 0 & 0 \\
\hline \multicolumn{3}{|l|}{$B$-factors } \\
\hline Protein & 111 & 328 \\
\hline Ligand/ion & 77 & - \\
\hline Water & - & - \\
\hline \multicolumn{3}{|l|}{ R.m.s deviations } \\
\hline Bond lengths $(\AA)$ & 0.010 & 0.010 \\
\hline Bond angles $\left(^{\circ}\right)$ & 1.5 & 1.3 \\
\hline
\end{tabular}

$R$ factors of the model (Table 1). Because of the low-resolution nature of the data set, this structure was not refined further.

Overall structure of two-subunit PC deletion mutant. Contrary to the expectations of an $\alpha_{4} \beta_{4}$ holoenzyme, the structure revealed a $370 \mathrm{kD} \alpha_{2} \beta_{4}$ oligomer for the MfPC mutant, with one BC dimer and two CT dimers (Fig. 2a). The mutant holoenzyme has overall dimensions of $140 \times 130 \times 70 \AA$ and adopts a shape similar to the letter $\mathrm{U}$, strikingly different from the holoenzyme architecture of single-chain PCs (Fig. 1b). The BC dimer (Fig. 2b) forms the bottom and a CT dimer is on each arm of the U shape, leaving a large cavity in the middle (Fig. 2a).

The $\mathrm{BC}$ dimer possesses a two-fold axis of symmetry, with root mean square (r.m.s.) distance of $0.1 \AA$ for the equivalent $C \alpha$ atoms of the two domains. However, the two CT dimers do not follow this symmetry, and have different orientation and position relative to the BC domains (Supplementary Fig. 6). This asymmetry would disfavour the binding of a second $\mathrm{BC}$ dimer at the top of the U-shaped structure, which partly explains the $\alpha_{2} \beta_{4}$ stoichiometry observed in the structure. In addition, one of the CT dimers ( $\beta 3$ and $\beta 4$ ) has relatively poor electron density and high temperature factors.

Of the three BCCP domains that are observed in this mutant holoenzyme, two are situated between the $\mathrm{BC}$ and $\mathrm{CT}$ domains, near the bottom of the U-shaped structure (Fig. 2a). These two BCCP domains likely come from the two $\beta$ subunits ( $\beta 2$ and $\beta 4$ ) that are distal from the $\mathrm{BC}$ dimer, and may help stabilize the overall structure of the mutant holoenzyme. The third BCCP 

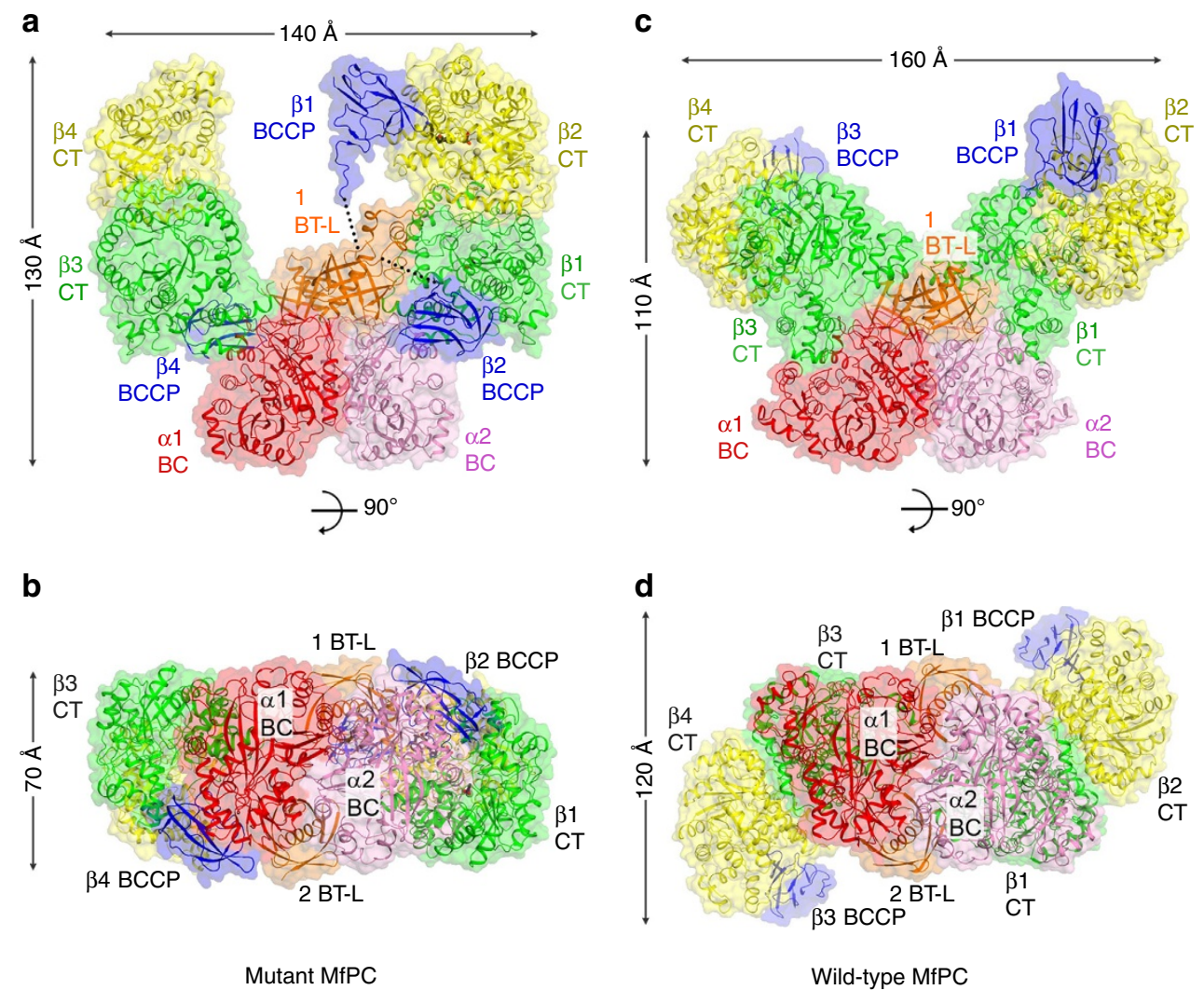

Figure 2 | Structures of MfPC holoenzyme. (a) Schematic drawing of the mutant MfPC $\alpha_{2} \beta_{4}$ holoenzyme structure, having the shape of the letter $U$ in this view. The domains are coloured according to Fig. 1a, except the BC domain of the second $\alpha$ subunit is coloured in pink. BT-L, BT-like domain. (b) Structure of the mutant MfPC holoenzyme viewed down the BC domain dimer, along the arrow of $\mathbf{a}$. (c) Structure of wild-type MfPC holoenzyme, having the shape of a butterfly in this view. (d) Structure of the wild-type MfPC holoenzyme viewed down the BC domain dimer, along the arrow of $\mathbf{c}$. The two distal $\beta$ subunits ( $\beta 2$ and $\beta 4$ ) are splayed away from each other, and other differences to the mutant MfPC holoenzyme are also clearly visible by comparing with $\mathbf{b}$.

domain is located in the active site of the distal $\beta$ subunit with good electron density $(\beta 2)$, and its biotin is well ordered (Supplementary Fig. 5), but it comes from a proximal $\beta$ subunit (likely $\beta 1$ ). Therefore, the BCCP domains are extensively swapped in the holoenzyme.

We assigned the BCCP in the CT active site as coming from the other proximal $\beta$ subunit based on considering the distances of the gaps. To provide evidence for this assignment, we separately purified wild-type $\alpha$ and $\beta$ subunits, along with A49T and K581A mutant $\beta$ subunits, which would inactivate the CT and BCCP domains, respectively (please see section below on structure-based mutations). The wild-type $\alpha$ subunit was then mixed separately with the three $\beta$ subunits to form the holoenzyme. As expected, the wild-type holoenzyme was fully active, while the holoenzymes containing the A49T or K581A mutant $\beta$ subunit had 1 and $2 \%$ of wild-type activity, respectively. We then diluted and mixed the two $\beta$ subunit mutants together in a 1:1 ratio, allowing the formation of homo and hetero mutant $\beta$ subunit dimers, before adding the $\alpha$ subunit to form a mixed population of hybrid mutant holoenzymes. If a BCCP domain can only visit the CT active site in the same $\beta$ subunit during catalysis, we would expect no recovery of activity. However, if a BCCP domain visits the active site in the other $\beta$ subunit during catalysis, about $\sim 25 \%$ of wild-type activity should be recovered. In support of the latter model and the assignments of BCCP in the structure, $29 \%$ of wild-type activity was recovered when the $\beta$ subunit mutants were combined.
Overall structure of wild-type two-subunit PC. The structure of the wild-type MfPC confirmed that the two-subunit PC holoenzyme has $\alpha_{2} \beta_{4}$ stoichiometry (Fig. 2c). However, the CT dimers moved closer to the $\mathrm{BC}$ dimer and the two proximal $\beta$ subunits moved close to each other as well, indicating that the contact between the $\mathrm{BC}$ and $\mathrm{CT}$ domains is flexible. Consequently, there is no space between the $\mathrm{BC}$ and $\mathrm{CT}$ domains to accommodate a BCCP domain and the large cavity in the centre of the mutant holoenzyme is absent as well. The wild-type holoenzyme instead has an overall shape of a butterfly, with overall dimensions of $160 \times 110 \times 120 \AA$ (Fig. 2 c). The two distal $\beta$ subunits are splayed away from each other (Fig. 2d), which would further disfavour the binding of a second $\mathrm{BC}$ dimer.

Compared with the single-chain PCs, the CT dimers in MfPC (Fig. 2d) are rotated by almost $90^{\circ}$ relative to those in SaPC (Fig. 1c and Supplementary Fig. 6). Therefore, the difference in the architecture of the two-subunit PC holoenzyme is not due simply to the removal of a $\mathrm{BC}$ dimer from one corner of the single-chain PC tetramer.

On the basis of the single-chain PC structures, we expected that the $\mathrm{B}$ domain of $\mathrm{BC}$ would not have interactions with other regions of the protein. However, the $B$ domain in the wild-type MfPC structure has some contact with the proximal $\beta$ subunit (Fig. 2c), due to the large differences in the orientation of the CT dimer compared with the single-chain PCs. The loss of this $\mathrm{BC}-\mathrm{CT}$ interaction in the mutant protein may explain why the orientation of the CT dimers differs from that in the wild-type enzyme. 
The structures of the individual $\mathrm{BC}$ and $\mathrm{CT}$ dimers of MfPC are largely similar to those of the single-chain PCs (Supplementary Fig. 7). The structure of the BC dimer is actually more similar to that of SaPC in complex with $\mathrm{CoA}^{10}$. The $\mathrm{C}$-terminal helix is disordered in the structure of the $\mathrm{BC}$ subunit of $A$. aeolicus $\mathrm{PC}^{19}$.

A BT-like structure in two-subunit PC. As in the single-chain PCs, there are few direct contacts between the BC and CT dimers in MfPC. Instead, interactions between the $\alpha$ and $\beta$ subunits are mediated primarily through a helix at the C-terminal end of an $\alpha$ subunit (residues 454-472, outside of the BC domain) and a four-stranded anti-parallel $\beta$-sheet (residues 470-512) in the CT-BCCP linker of a $\beta$ subunit, equivalent to the PT domain in the single-chain PCs (Fig. 1a), with r.m.s. distance of $3.7 \AA$ for 56 equivalent $\mathrm{C} \alpha$ atoms between the MfPC and SaPC structures (Supplementary Fig. 7).

Surprisingly, the structure shows that the $\beta$-sheet from the second subunit of the CT dimer wraps around the other face of the helix (Fig. 2a), giving rise to a domain with a central $\alpha$-helix surrounded by an eight-stranded up-down $\beta$-barrel (Fig. $3 \mathrm{a}$ and Supplementary Fig. 5). The overall structure of this domain is therefore remarkably similar to that of the BT (BC-CT interaction) domain found in the structures of propionyl-CoA carboxylase (PCC ${ }^{20}, 3$-methylcrotonyl-CoA carboxylase $e^{21}$ and $\mathrm{ACC}^{22}$. The r.m.s. distance is $3.2 \AA$ for 88 equivalent $\mathrm{C} \alpha$ atoms between MfPC and the PCC BT domain (Fig. 3b), even though the latter has a longer central helix and a 'hook' linking the helix and the first strand of the barrel that is important for interactions in the PCC holoenzyme. The stoichiometry of this BT-like domain is $\alpha_{1} \beta_{2}$, and the formation of this domain is likely the primary determinant for the $\alpha_{2} \beta_{4}$ stoichiometry of the MfPC holoenzyme. While the BT-like domain has essentially no sequence identity with the PT domain of single-chain PCs, there is a high degree of sequence conservation of the BT-like domain among all bacterial two-subunit PCs (Supplementary Figs 2 and 3), strongly suggesting that these enzymes share a common $\alpha_{2} \beta_{4}$ subunit composition.

An important difference between the two-subunit and singlechain PCs is that the helix in the PT domain of single-chain PCs is amphipathic, with the hydrophilic side exposed to the solvent in the holoenzyme (Fig. 3c,d). In contrast, the central helix of the BT-like domain in two-subunit PCs is entirely hydrophobic, consisting primarily of alanine residues (Fig. 3e), and is completely shielded from solvent by the $\beta$-barrel structure (Fig. 3f). This is similar to the BT domain in PCC and 3-methylcrotonyl-CoA carboxylase.

A highly conserved 476-HGE-478 motif in a $\beta$-hairpin of the $\mathrm{BT}$-like domain interacts with residues at the $\mathrm{BC}$ dimer interface (Fig. 4a). Additional conserved residues at this interface include Asp502 and Glu507 in the BT-like domain. However, these residues are not conserved in single-chain PCs (Supplementary Fig. 3). Instead, this region is equivalent to the binding site for the adenine diphosphate portion of acetyl-CoA in single-chain PCs $(\text { Fig. } 4 \mathrm{~b})^{8-10}$. Especially, the side chain of His 476 occupies the same position as the adenine base of acetyl-CoA, and forms an equivalent hydrogen bond with the carbonyl of Ala45 (Ala47 in SaPC). The Glu478 side chain is located near the $3^{\prime}$ phosphate of acetyl-CoA, and they both interact with residues in the opposite $\mathrm{BC}$ monomer. This is consistent with the fact that the $\mathrm{BC}$ dimer in MfPC is more similar to the CoA complex of SaPC (Supplementary Fig. 7), even though there is a significant difference in the position of the BT-like domain relative to the PT domain of single-chain PCs (Fig. 4b). This may also explain why two-subunit PCs are not sensitive to acetyl-CoA, as the binding site is occupied by a protein segment from the $\beta$ subunit. The conformational change in the SaPC BC dimer induced by acetyl-CoA binding has also been linked to the activation of the enzyme ${ }^{10}$. Thus, the conformation of MfPC BC dimer induced by the 476-HGE-478 motif may be responsible for the high $k_{\text {cat }}$ value observed for this enzyme (Supplementary Fig. 4).

Further evidence for the $\boldsymbol{\alpha}_{2} \boldsymbol{\beta}_{\mathbf{4}}$ stoichiometry. The $\alpha_{2} \beta_{4}$ stoichiometry of two-subunit PCs is unusual in light of the structures of the single-chain PCs along with the fact that all other biotin-dependent carboxylases studied to date have a 1:1 ratio between their BC and CT domains. Therefore, we sought further evidence in support of this stoichiometry.

We confirmed that the $\alpha_{2} \beta_{4}$ complex is not limited just to MfPC, as all the bacterial two-subunit PCs that we were able to express and purify from E. coli migrated at nearly the same position as MfPC on a gel filtration column, substantially later than that for the single-chain SaPC (Fig. 5a), and an SDS-polyacrylamide gel electrophoresis (SDS-PAGE) gel showed that the two-subunit PCs have the same ratio of $\alpha$ to $\beta$ subunits (Fig. 5b). The 1:2 stoichiometry of the subunits is further confirmed by running known amounts of the individual subunits in an SDS-PAGE gel (Fig. 5c). To address the possibility that expression of the $\beta$ subunit was somehow favoured over the $\alpha$ subunit in $E$. coli, we introduced a separate plasmid expressing only the $\alpha$ subunit along with the bicistronic plasmid expressing both $\alpha$ and $\beta$ subunits. After purification, the complex was identical to that obtained from the bicistronic plasmid only, migrating at the same position on the gel filtration column and showing excess $\beta$ by SDS-PAGE.

As further evidence in support of the $\alpha_{2} \beta_{4}$ stoichiometry of two-subunit PCs, we carried out analytical ultracentrifugation experiments with MfPC (wild-type and the deletion mutant) and PaPC, and we used single-chain SaPC as a control. All three two-subunit PC samples gave molecular masses of $\sim 340 \mathrm{kD}$ $\left(\sim 11.5 \mathrm{~S}\right.$ ), consistent with the $\alpha_{2} \beta_{4}$ stoichiometry (Fig. $5 \mathrm{~d}$ and Supplementary Fig. 8). In comparison, SaPC gave a molecular mass of $\sim 570 \mathrm{kD}(14.8 \mathrm{~S})$, consistent with a homo-tetramer as we found earlier ${ }^{25}$.

We then purified the $\alpha$ and $\beta$ subunits of MfPC separately and mixed them together in different molar ratios, followed by analytical gel filtration. Mixing of $\alpha$ and $\beta$ in 1:2, 1:1 or 2:1 molar ratios did not change the composition of the complex as they all migrated similarly on the gel filtration column (Fig. 5e) and showed excess $\beta$ over $\alpha$ by SDS-PAGE (Fig. 5f). Moreover, when $\alpha$ and $\beta$ subunits were mixed in a 1:1 molar ratio, only half of the total $\alpha$ formed the complex and the rest migrated as free $\alpha$ on the gel filtration column. Only when $\alpha$ and $\beta$ were mixed in a 1:2 molar ratio was all of the $\alpha$ subunit absorbed into the complex.

While it has been generally accepted that the bacterial two-subunit PCs form an $\alpha_{4} \beta_{4}$ complex $^{12}$, earlier biochemical characterizations of these enzymes from several species actually produced a range of estimates for the molecular weight and subunit composition ${ }^{17,26,27}$. Studies of the two-subunit PC from A. vinelandii, Pseudomonas citronellolis and P. fluorescens reported a molecular weight of $\sim 300 \mathrm{kD}$ based on gel filtration and sedimentation experiments, and these groups proposed an $\alpha_{2} \beta_{2}$ subunit stoichiometry ${ }^{17,26,27}$. On the other hand, another study on the PC enzyme from P. citronellolis reported a molecular weight of $\sim 500 \mathrm{kD}$ based primarily on gel filtration analysis, and this group proposed an $\alpha_{4} \beta_{4}$ subunit composition ${ }^{12}$. However, $\alpha_{4} \beta_{4}$ subunit ratios of $1: 1.7$ and 1:1.18 were found based on two different protocols for Coomassie blue staining of SDS gels, which is inconclusive between 1:1 and 1:2 ratios of subunits. On the basis of SDS-PAGE gels, PaPC appears to have an excess of 
a

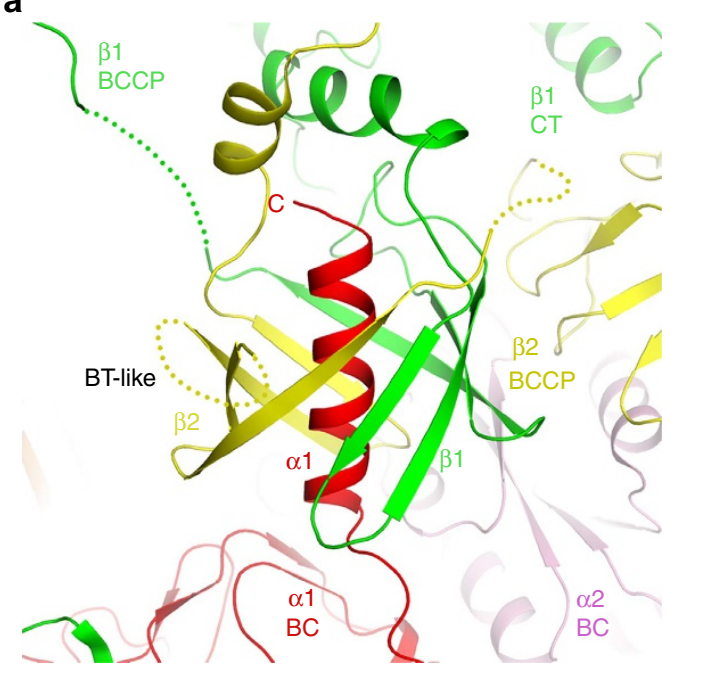

C

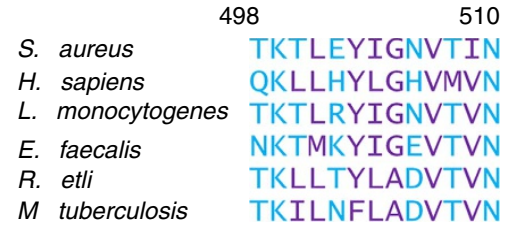

b

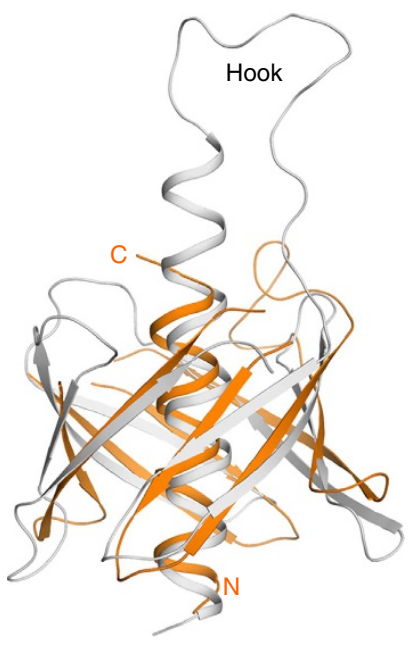

e

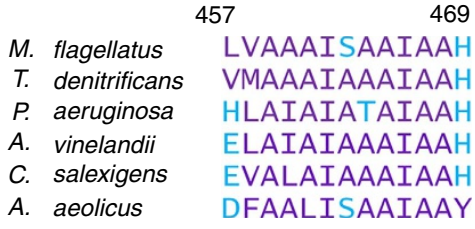

d

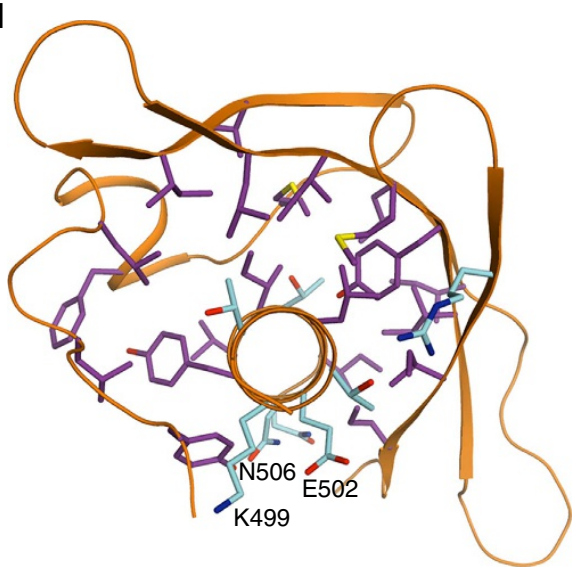

f

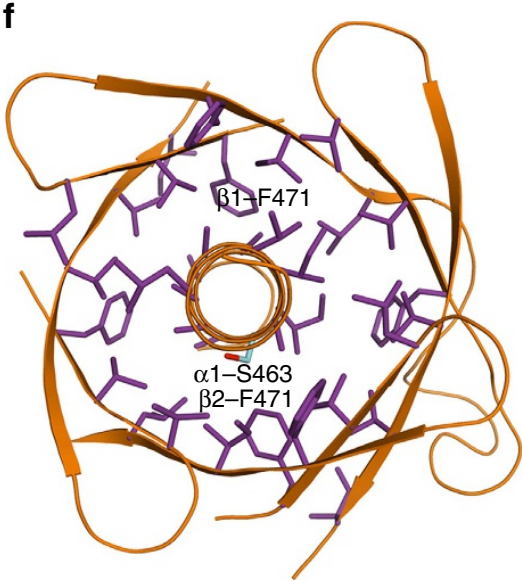

Figure 3 | A BT-like domain in the structure of two-subunit PC. (a) Schematic drawing of the BT-like domain in MfPC. The helix comes from the $\alpha$ subunit (red), and the two four-stranded $\beta$-sheets come from two separate $\beta$ subunits (green and yellow). Each subunit is given a different colour to highlight the origins of the protein segments in the BT-like domain. (b) Overlay of the structures of the BT-like domain of MfPC (orange) and the BT domain of PCC (grey) ${ }^{20}$. (c) Sequence alignment of the residues in the $\alpha$ helix of the PT domain in single-chain PCs. Hydrophobic residues are coloured purple, and hydrophilic ones in cyan. (d) The $\alpha$ helix of the PT domain in SaPC is amphipathic, with half of its surface having hydrophilic residues and exposed to the solvent. Hydrophobic side chains are coloured in purple, and hydrophilic ones in cyan. (e) Sequence alignment of the residues in the $\alpha$ helix of the BT-like domain in two-subunit PCs, at the C-terminal end of the $\alpha$ subunit. (f) The $\alpha$ helix of the BT-like domain in MfPC is hydrophobic, completely surrounded by the $\beta$-barrel and having mostly small side chains.

$\beta$ subunit when it is expressed aerobically in Pseudomonas ${ }^{11}$. The wide range of these estimates is likely due to the limitations of using gel filtration to determine molecular weights, particularly for large proteins with extended shapes.

BCCP movement during catalysis. The two-subunit PCs are unique among the biotin-dependent carboxylases in that they have a 1:2 ratio of $\mathrm{BC}: \mathrm{CT}$ active sites. In both the wild-type and mutant structures, a BCCP domain is found only in the active site of the distal CT domain (Fig. 2a,c). This distal CT active site is located $70 \AA$ away from the nearest BC active site (Supplementary
Fig. 9), a distance which is within the range found in other biotindependent carboxylases, and which supports a swinging-domain model for catalysis ${ }^{2}$. The proximal CT domain active site is accessible to BCCP in the mutant structure, but inaccessible in the wild-type structure due to clashes with the $\mathrm{BC}$ dimer (Supplementary Fig. 9). This suggests the possibility that only the distal CT domain is catalytically active. In this model, one $\beta$ subunit would provide the active CT domain, while the other $\beta$ subunit provides the active BCCP domain.

We cannot rule out an alternative model in which conformational changes in the wild-type enzyme allow both CT domains to be involved in catalysis. The CT active site of the proximal $\beta$ 
a

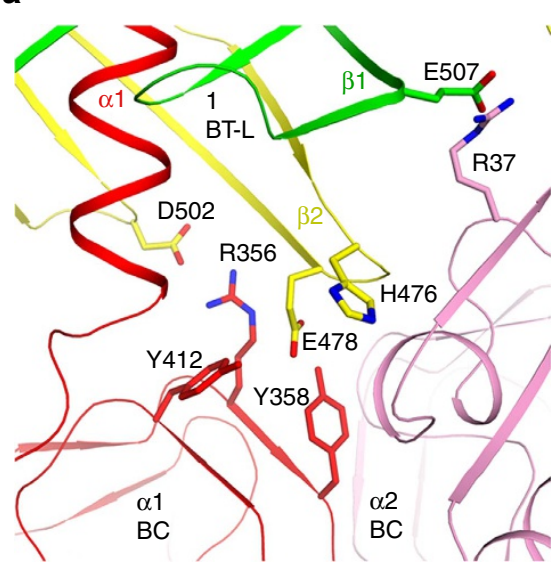

b

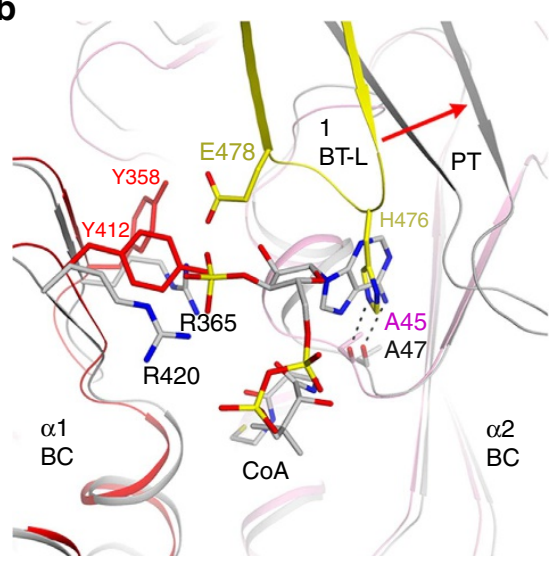

C

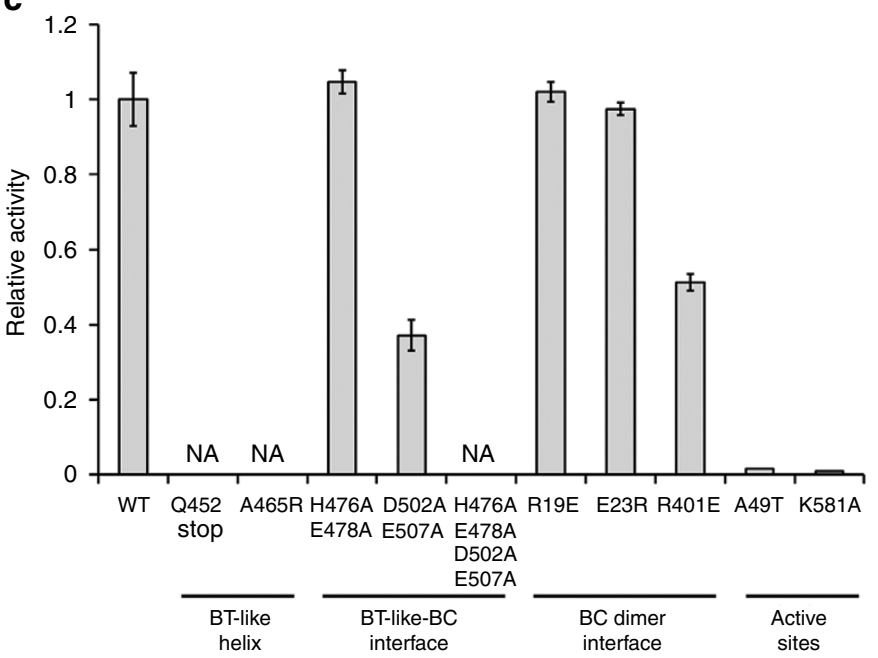

d

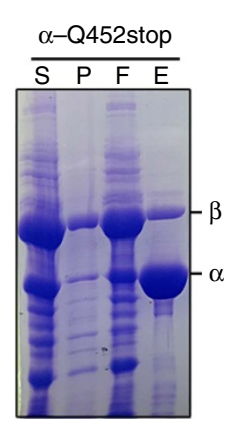

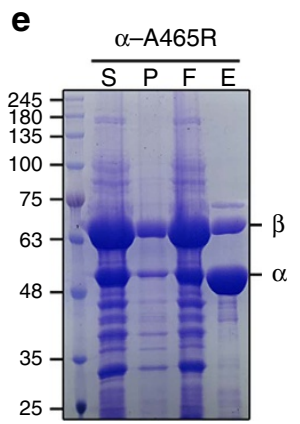

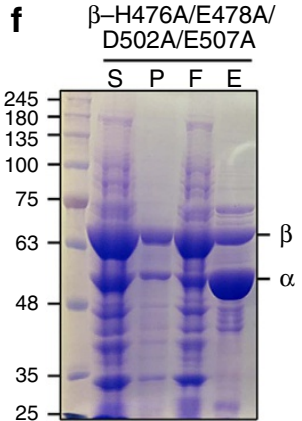

Figure 4 | Mutagenesis studies to assess the structural observations. (a) Interactions between the BT-like domain and the BC dimer at the holoenzyme interface. (b) Overlay of the structure of MfPC (in colour) and SaPC (in grey) in complex with CoA (grey for carbon atoms) ${ }^{10}$. The red arrow indicates the difference in position of the BT-like domain in MfPC and the PT domain in SaPC. (c) Catalytic activities of wild-type and mutant MfPCs. The pyruvate concentration is at $20 \mathrm{mM}$. The error bars represent the s.d. from three independent measurements. NA, no activity observed under the condition tested. (d) Deletion of the $\alpha$ helix of the BT-like domain, or mutation of one of its Ala residues (A465R), abolished the formation of the MfPC holoenzyme. The two subunits were co-expressed in $E$. coli, with the $\alpha$ subunit carrying a His-tag. E, eluate from the Nickel column; F, flow-through of the Nickel column; $\mathrm{P}$, pellet; $\mathrm{S}$, soluble fraction. (e) Mutation of an Ala residue (A465R) in the helix of the BT-like domain abolished the formation of the MfPC holoenzyme. (f) A quadruple mutation in the interface between the BT-like domain and BC blocked holoenzyme formation.

subunit is likely $\sim 40 \AA$ away from the same BC active site that will be accessed by the distal $\beta$ subunit (Supplementary Fig. 9), and therefore both BCCP domains of a $\beta$ dimer likely visit the same BC active site during catalysis. It is not clear from the structures whether there would be coordination between the two BCCP domains in their access to this active site. It could be possible that while one $\mathrm{BCCP}$ domain is in the $\mathrm{BC}$ active site, the other BCCP domain is in the CT active site.

Structure-based mutations block holoenzyme formation. We introduced mutations in MfPC based on the structural observations, and tested their effects on catalysis (Fig. 4c) and/or holoenzyme formation. As expected, deleting the last helix of the $\alpha$ subunit with the Q452stop mutant, in the centre of the BT-like domain, abolished the formation of the holoenzyme (Fig. 4d). In addition, mutating one of the alanine residues in this helix to arginine (A465R) also abrogated the formation of the holoenzyme, confirming that it is necessary for the helix to have small hydrophobic residues to form the intact BT-like domain (Fig. 4e). Both mutants are catalytically inactive as well (Fig. 4c). We then assessed the function of the conserved residues at the interface between the BC- and BT-like domains (Fig. 4a) by generating the double mutants H476A/E478A and D502A/E507A. Both of these double mutants still formed a stable holoenzyme, and the H476A/E478A mutant was fully active while the D502A/E507A double mutant retained $40 \%$ of wild-type activity (Fig. 4c). However, the H476A/E478A/D502A/E507A quadruple mutation completely abolished holoenzyme formation (Fig. 4f) and catalytic activity (Fig. 4c), consistent with the structural observations and implicating this interface as a key region for stabilizing the holoenzyme.

The BC dimer interface is similar to that observed in SaPC (Supplementary Fig. 7), as well as the BC subunit of E. coli ACC $^{28,29}$. Mutational studies of SaPC demonstrated that a single point mutation (K442E) at the BC dimer interface is sufficient to disrupt tetramerization of the enzyme and abolish catalytic activity $^{25}$. We introduced the equivalent mutation (R401E) in the $\alpha$ subunit of MfPC, but found that it still formed the $\alpha_{2} \beta_{4}$ complex and had only a $\sim 50 \%$ reduction in catalytic activity compared with wild-type MfPC (Fig. 4c). We tested two additional BC dimer interface mutants, R19E and E23R. In SaPC, the R19E mutant forms a tetramer but does not have catalytic activity, while E23R formed aggregates. The R19E and E23R 
a

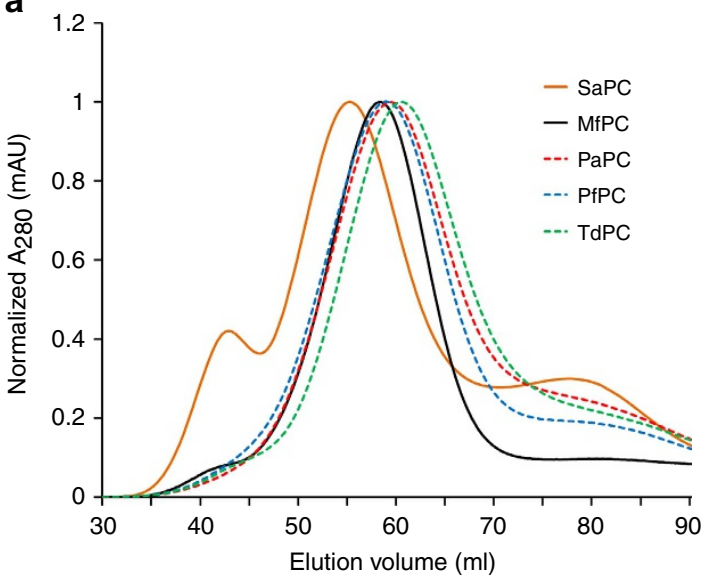

b

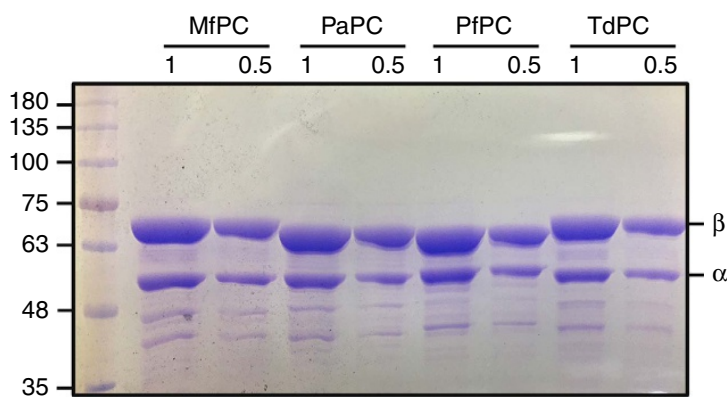

e

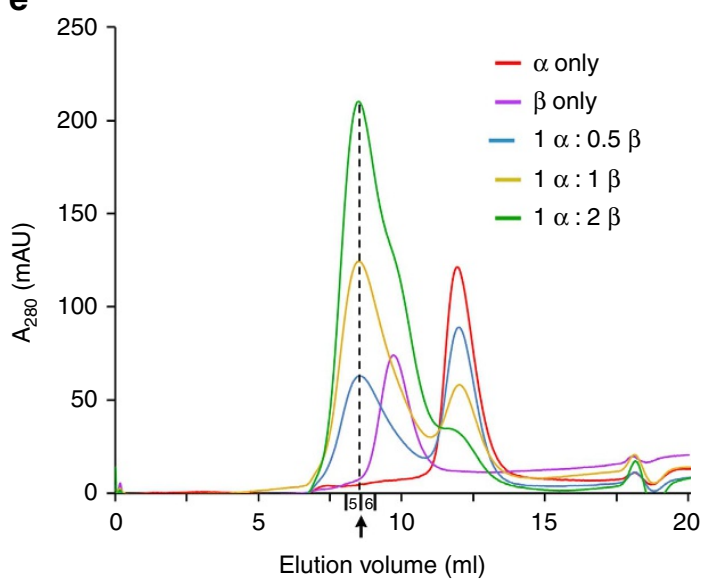

f
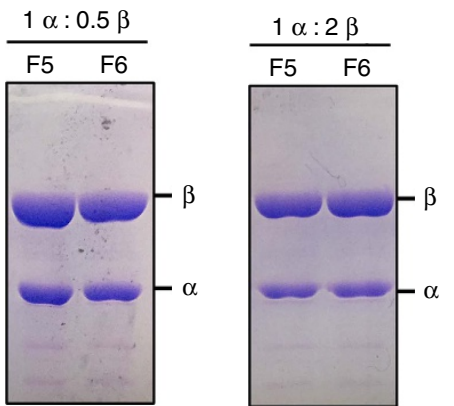

c Standard with isolated MfPC $\alpha$ and $\beta$ subunits

Purified

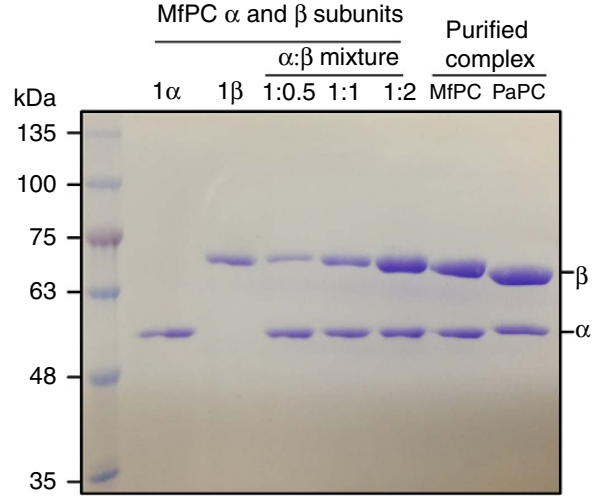

d

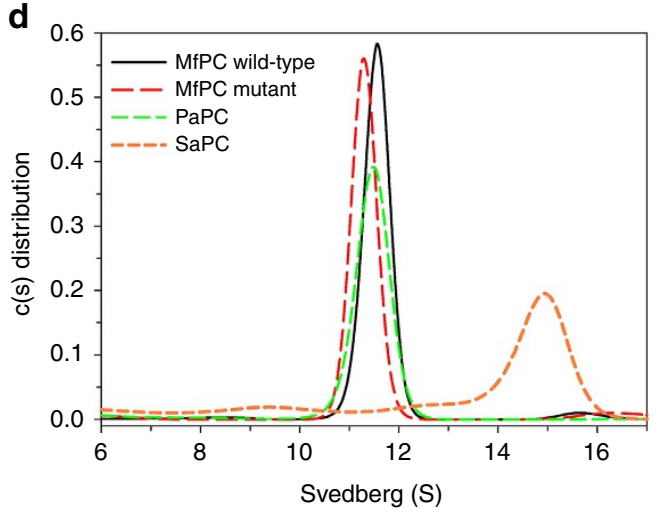

Figure 5 | Experiments to confirm $\boldsymbol{\alpha}_{\mathbf{2}} \boldsymbol{\beta}_{\mathbf{4}}$ stoichiometry of two-subunit PCs. (a) Gel filtration profiles for two-subunit PCs (Mf, M. flagellates; Pa, P. aeruginosa; Pf, P. fluorescens; Td, T. denitrificans) and the single-chain SaPC. (b) Coomassie-stained SDS-PAGE gel with purified two-subunit PCs from different species. Two lanes were run for each protein, with twice as much protein loaded in the first lane. The band for the $\alpha$ subunit in this lane is roughly the same as that for the $\beta$ subunit in the second lane, consistent with the 1:2 stoichiometry. (c) SDS-PAGE gel with known amounts of $\alpha$ and $\beta$ subunits and their mixtures, indicating that the purified holoenzyme has 1:2 stoichiometry. The $\beta$ subunit in the MfPC holoenzyme sample is slightly smaller than the $\beta$ subunit sample alone as it does contain a His tag. (d) Size distribution of PCs in solution at a concentration of $0.5 \mathrm{mg} \mathrm{ml}^{-1}$, based on the best-fit results by the continuous size distribution analysis. Two-subunit PCs (MfPC wild-type and deletion mutant, and PaPC) showed a similar major species of $S=11.5$, while single-chain SaPC showed a tetrameric species of $S=14.8$. (e) Gel filtration profiles from mixing experiments of different ratios of MfPC $\alpha$ and $\beta$ subunits. The vertical dashed line indicates the elution volume of the complex that is formed on mixing. (f) Coomassie-stained SDS-PAGE gels of peak fractions collected from two different mixing experiments (F5 and F6 indicates collected fractions 5 and 6, respectively, indicated in e).

MfPC mutants were also able to form the holoenzyme and did not differ markedly from the wild-type enzyme in catalytic activity (Fig. 4c). Overall, these results indicate that the MfPC complex is more robust to mutations at the $\mathrm{BC}$ dimer interface compared with SaPC. The single-chain PC monomers are held together in the tetramer primarily by the $\mathrm{BC}$ and $\mathrm{CT}$ dimer interfaces (Fig. 1b). The additional inter-subunit interactions mediated by the BT-like domain in MfPC may allow for the complex to better tolerate these $\mathrm{BC}$ interface mutations and retain catalytic activity. The $\alpha$ subunit of MfPC alone is a monomer in solution (Fig. 5e), and the $\mathrm{BC}$ domain from $\mathrm{SaPC}$ alone is also a monomer and catalytically inactive ${ }^{25}$, indicating that additional interactions within the holoenzyme are necessary to form a stable $\mathrm{BC}$ dimer in these PC enzymes.

We also created the A49T mutant of MfPC, equivalent to the disease-causing A610T mutant of HsPC. This residue is located in the CT active site (Supplementary Fig. 9), and the bulkier Thr side chain is expected to interfere with biotin binding ${ }^{10}$. The A49T 
mutant had essentially no catalytic activity (Fig. 4c). In addition, blocking biotinylation of the enzyme by mutating the Lys581 residue in BCCP to Ala also abolished catalysis, as expected (Fig. 4c).

Colony morphogenesis of $\boldsymbol{P}$. aeruginosa PC mutant strains. We then assessed the functional importance of the two-subunit PC in the human pathogen $P$. aeruginosa ( $\mathrm{PaPC})$. $\mathrm{PaPC}$ has high sequence identity with MfPC (67\% identity overall, 74\% BC, $72 \%$ CT, 53\% BT-like and 62\% BCCP), and is likely to have the same $\alpha_{2} \beta_{4}$ stoichiometry and architecture based on our gel filtration and sedimentation analysis (Fig. 5a,d). We created a markerless deletion of the PC operon, $\triangle P C$ ( $\triangle P A 14$ 71720-PA14_71740), and a series of point mutants that would allow us to assess the functional importance of key residues identified in the structure and that are conserved between M. flagellatus and P. aeruginosa. The growth profiles of these strains were analysed in a defined medium containing either succinate (a C4 compound), pyruvate (C3) or glucose (C6) as the sole carbon source. We did not observe any major effects when growth of the mutant strains was compared with that of the wild type on succinate (Fig. 6a). While all strains showed overall decreased growth on pyruvate, a subset of the strains, including $\triangle P C$, exhibited severe growth defects relative to the wild type (Fig. $6 \mathrm{~b}$ ). The K451stop mutation in the $\alpha$ subunit (equivalent to Q452 in MfPC), which would delete the helix of the BT-like domain and disrupt holoenzyme formation, and the K572A mutation in the $\beta$ subunit (equivalent to K581 in
MfPC), which would abolish biotinylation, led to growth defects in pyruvate that were similar to that of the $\triangle P C$ deletion mutant. The A55T mutation in the $\beta$ subunit (equivalent to A49 in MfPC), which reduces PC activity by 50 -fold and would interfere with biotin binding to the CT active site, exhibited growth on pyruvate that was similar to the wild type. Finally, the growth defect of $\triangle P C$ was rescued when the PC operon was placed back into the endogenous locus (Fig. 6a). These phenotypes were recapitulated on glucose, though all strains showed better growth overall on this carbon source. Defective growth of mutants with impaired PC activity on C3 and C6 (but not C4) carbon sources confirms earlier findings ${ }^{14}$.

While culturing in well-aerated, homogenous liquid medium has been a traditional system for assessing microbial growth phenotypes, it does not represent the primary mode of growth outside the laboratory for most microbes. In non-laboratory habitats, microbes typically grow as communities of densely packed cells held together by a self-produced matrix. Cells in matrix-encased assemblages often exhibit unique metabolisms due to resource limitation. To examine a possible role for PC in $P$. aeruginosa growth under such conditions, we adapted defined media for use in two additional models that assess the production of extracellular matrix: (i) a pellicle assay, in which liquid cultures are incubated without shaking and biofilms develop at the air-liquid interface; and (ii) a colony morphology assay, in which cell suspensions are spotted on agar-solidified media containing the dyes Congo red and Coomassie blue and matrix production is required for the formation of wrinkle structures on the colony
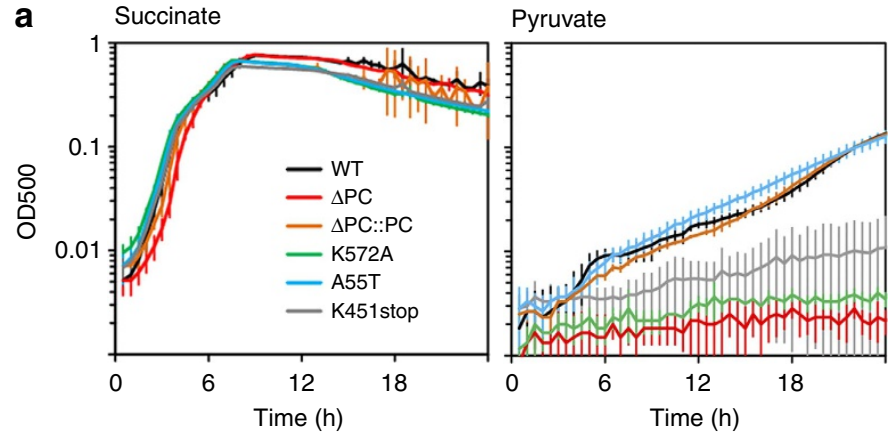

Glucose

b

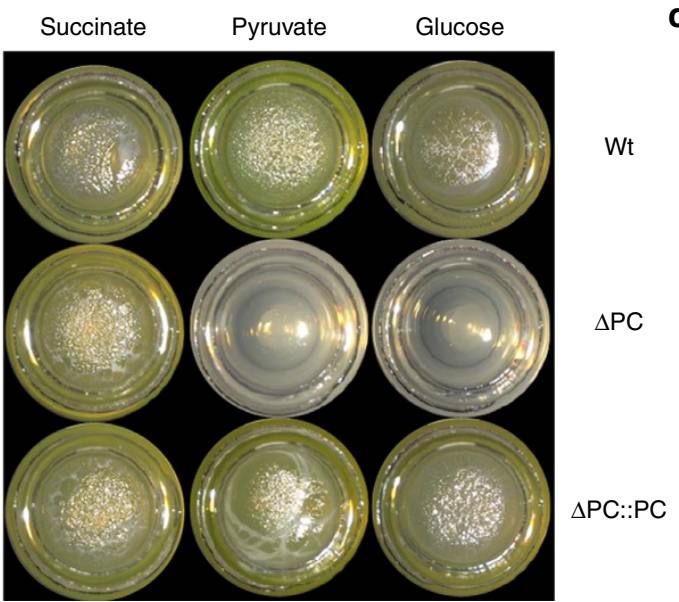

C
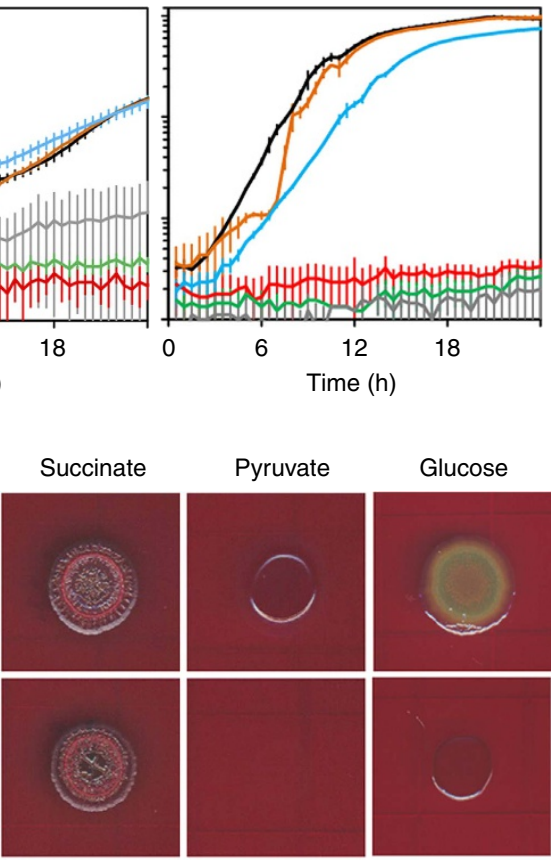

Glucose
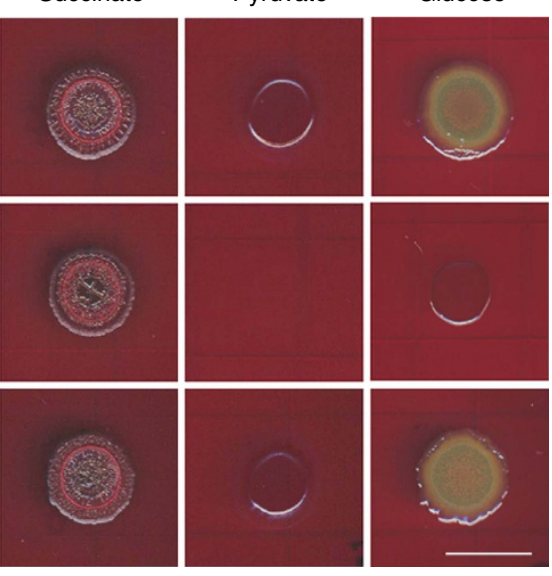

Figure 6 | The two-subunit PaPC is required for growth on selected carbon sources. (a) Shaken liquid-culture growth of wild-type $P$. aeruginosa PA14, $\triangle P C$, the $P C$-complemented strain and various site-specific mutants in a defined medium containing $20 \mathrm{mM}$ succinate, pyruvate or glucose as the sole carbon source. Each growth curve represents the average of three biological replicates. Error bars denote s.d. (b) Pellicles formed by P. aeruginosa PA14, $\triangle P C$, and the PC-complemented strain on a defined medium containing succinate, pyruvate, or glucose as the sole carbon source. (c) Colony morphology of P. aeruginosa PA14, $\triangle P C$ and the PC-complemented strain on a defined medium (supplemented with 1\% agar, Congo red and Coomassie blue) containing succinate, pyruvate or glucose as the sole carbon source. Images depict day 3 of colony development. 
a

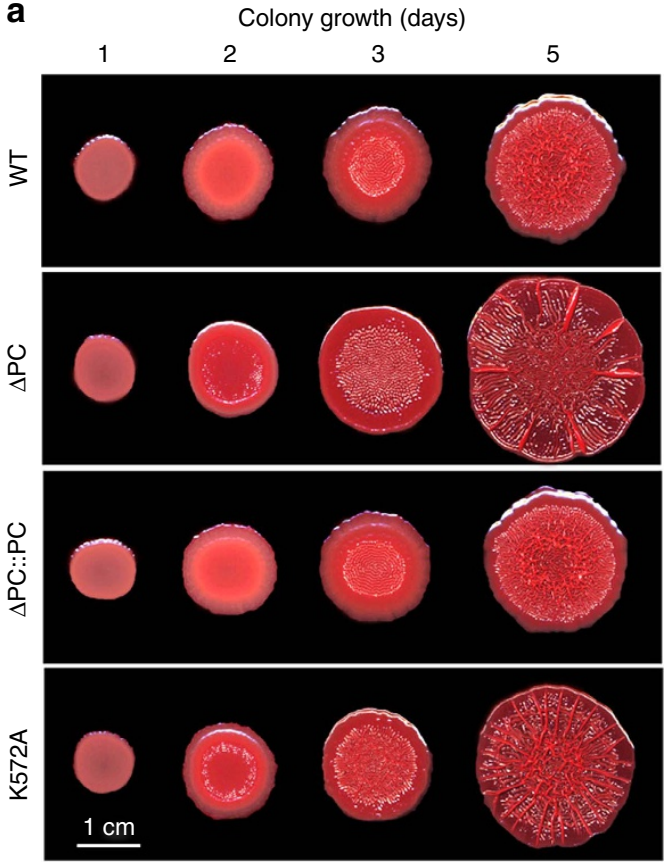

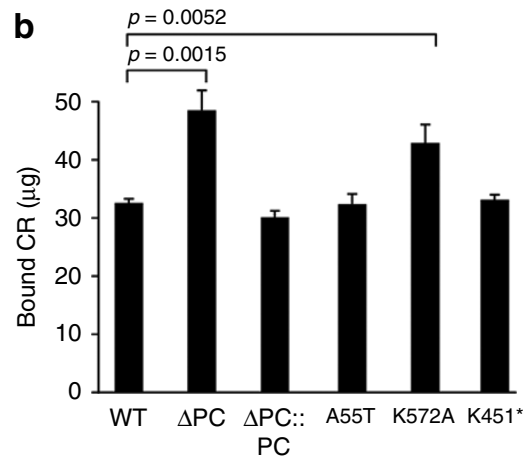

C

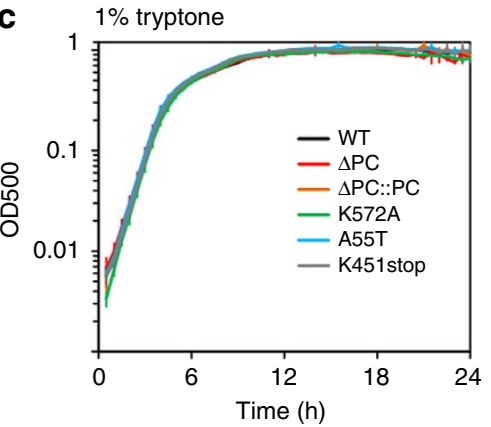

Figure 7 | PaPC dysfunction leads to increased matrix production and altered colony morphology. (a) Colony morphology of wild-type $P$. aeruginosa PA14, $\triangle P C$, the PC-complemented strain and the K572A mutant on 1\% tryptone, 1\% agar (supplemented with Congo red and Coomassie blue).

(b) Quantification of Congo red binding, a proxy for matrix production, for P. aeruginosa PA14, $A P C$, the PC-complemented strain and various site-specific mutants grown on $1 \%$ tryptone, $1 \%$ agar with no added dyes. Colonies were grown for 3 days before they were collected for the Congo red binding assay. (c) Shaken liquid-culture growth of $P$. aeruginosa PA14, $\triangle P C$, the PC-complemented strain and various site-specific mutants in $1 \%$ tryptone. Each growth curve represents the average of three biological replicates. Error bars denote s.d.

surface $^{30,31}$. All strains formed robust pellicles and colony structures when succinate was supplied as the carbon source, with the $\triangle P C$ showing similar morphologies to those of the wild type. However, when glucose or pyruvate was provided as the carbon source, the $\triangle P C$ mutation prevented growth (Fig. 6b,c).

The growth phenotypes we observed in the pellicle and colony morphology assays recapitulated the results for growth in aerated liquid cultures. However, utilization of complex medium (1\% tryptone) revealed a PC-dependent phenotype specific to matrix production in colonies (Fig. 7a,b) (since we did not observe robust pellicle formation for wild-type PA14 in standing cultures of $1 \%$ tryptone medium, we could not test for altered pellicle formation under these conditions). In the colony morphology assay, the wild type initially produces a smooth colony, which forms wrinkle structures in the centre starting on day 3 (Fig. 7a). By day 5, the wrinkles have spread over the colony, but a region of smooth morphology remains at the colony edge. We found that, in contrast, $\triangle P C$ and $\mathrm{K} 572 \mathrm{~A}$ colonies begin to wrinkle on day 2 and exhibit a flatter morphology with several exaggerated wrinkles in a 'spoke' formation, which is reminiscent of morphologies that arise during electron acceptor-limited conditions ${ }^{32}$. Colonies formed by all other mutants exhibited morphologies that were similar to the wild type. Quantification of the cationic exopolysaccharide Pel, a major component of the PA14 biofilm matrix ${ }^{33}$, showed that, indeed, increased matrix production is associated with enhanced colony wrinkling (Fig. 7a,b). Wild-type colony morphology and matrix production were restored in the $\triangle P C$ complementation strain (Fig. 7a). Interestingly, colony morphology and liquid-culture growth phenotypes did not correlate. Though the $\triangle P C$ and K572A mutants showed altered colony morphogenesis on agar-solidified $1 \%$ tryptone, they were unaffected in shaken liquid-culture growth on this medium. Further, while both the K572A and K451stop mutants exhibit defects when grown on defined media containing pyruvate or glucose as the sole carbon source, only K572A shows altered development in the colony morphology assay on tryptone. The colony morphology assay therefore reveals PC-dependent metabolic disruptions that specifically affect a matrix-encased, structured community.

\section{Discussion}

The central helix at the C-terminal end of the $\alpha$ subunit is not present in the $\mathrm{BC}$ subunit of bacterial multi-subunit $\mathrm{ACC}^{34}$. In fact, many of the $\mathrm{PC} \alpha$ subunits are mis-annotated as $\mathrm{BC}$ subunits of ACC in the sequence database, and the presence of this C-terminal helix can be used to readily distinguish between them. In addition, the multi-subunit ACC and the two-subunit PC are both present in several bacterial species, including $P$. aeruginos ${ }^{2}$, and this C-terminal helix may ensure that the correct $\alpha$ subunit is incorporated into the complex in vivo.

The PC enzymes from archaea are also separated into $\alpha$ and $\beta$ subunits 35,36 , but they have several major differences from bacterial two-subunit PCs. The $\beta$ subunit of archaeal PCs is $\sim 570$ residues, significantly shorter than the 600-620 residues of bacterial two-subunit PCs. This is due to a large deletion in the region corresponding to the putative PT-/BT-like domain, such that only two $\beta$ strands are predicted. The $\alpha$ subunit of bacterial two-subunit PCs is generally $\sim 471$ residues and terminates precisely at the end of the BT-like domain $\alpha$ helix (Supplementary Fig. 2). In comparison, the $\alpha$ subunit of archaeal PCs has $\sim 500$ residues due to a much longer $\mathrm{C}$-terminal extension. In addition, while a stretch of small hydrophobic residues is present in the putative PT-/BT-like domain helix, it is much shorter than that in 
bacterial two-subunit PCs. Because of these significant differences, it is unclear if archaeal two-subunit PCs share the subunit stoichiometry and holoenzyme architecture of bacterial two-subunit PCs. Thus, it may be better to classify the bacterial and archaeal two-subunit PCs into two distinct categories.

The multifunctional biotin-dependent carboxylases likely arose through gene fusion of smaller mono-functional precursors. The structures of the homo-tetrameric and the two-subunit PCs give general insights into the mechanisms by which a protein can adapt to the gene fusion event, with the newly formed multidomain protein preserving essential functions while gaining new features. An important consequence of this gene fusion event in the case of PC would be the disruption of the $\alpha_{1} \beta_{2}$ stoichiometry of the BT-like domain, likely precipitating the drastic differences observed in holoenzyme architectures. However, the structures of the individual domains and the catalytic mechanism appear to be conserved between homo-tetrameric and two-subunit PCs. With the disruption of the BT-like domain, the new PT domain acquired additional functions, including tetramer stabilization and recognition of the allosteric activator acetyl-CoA. Intriguingly, acetyl-CoA binds to single-chain PCs in a conformation that mimics an important structural motif in the two-subunit PCs, suggesting that the development of acetyl-CoA regulation of PC might be linked to the structural rearrangements induced by the fusion. The various structures of biotin-dependent carboxylases have demonstrated that homologous domains can be arranged into remarkably different holoenzyme architectures, and that these distinct architectures have been tuned by evolutionary processes to perform distinct functions in cells.

Our in vivo results obtained with $P$. aeruginosa PA14 PC mutants confirm the role of $\mathrm{PaPC}$ during growth on pyruvate, a C3 compound, and support the model that this enzyme functions to replenish $\mathrm{C} 4$ intermediates thereby enabling TCA cycle function ${ }^{14}$. We also observed that impaired PC function led to increased matrix production and the development of flat colonies with pronounced wrinkle features on a medium containing tryptone as the carbon source. We have previously observed this phenotype under electron acceptor limitation ${ }^{32}$ and hypothesize that it is an adaptive community response to cellular redox imbalance. This suggests that the anaplerotic role of PC supports TCA cycle function and redox homeostasis during multicellular growth on complex carbon sources and may contribute to $P$. aeruginosa pathogenicity via this mechanism ${ }^{15}$.

\section{Methods}

Protein expression and purification. The $\alpha$ and $\beta$ subunits for PC from several bacterial species, including $M$. flagellatus, $P$. aeruginosa, $P$. fluorescens and Thiobacillus denitrificans were amplified from genomic DNA (American Type Culture Collection) and sub-cloned into the pCDFduet vector (Novagen). The $\alpha$ subunit was sub-cloned into MCS1 with an N-terminal hexa-histidine tag, and the $\beta$ subunit was sub-cloned into MCS2 with no tag. The internal B-domain deletion was made using overlapping PCR. Surface entropy reduction mutations were chosen based on the UCLA SerP server ${ }^{24}$. The individual $\alpha$ and $\beta$ subunits for $\mathrm{MfPC}$ were sub-cloned into pET28a with an $\mathrm{N}$-terminal hexa-histidine tag. All expression constructs were co-transformed into BL21 Star (DE3) cells along with a plasmid encoding the E. coli biotin ligase (BirA) gene.

The cells were cultured in Luria-Bertani (LB) medium with $50 \mathrm{mg} \mathrm{ml}^{-1}$ streptomycin and $35 \mathrm{mg} \mathrm{ml}^{-1}$ chloramphenicol, and were induced for $14 \mathrm{~h}$ with $1 \mathrm{mM}$ isopropyl- $\beta$-D-thiogalactosideat $20^{\circ} \mathrm{C}$. Before induction, $20 \mathrm{mg} \mathrm{l}^{-1}$ biotin and $10 \mathrm{mM} \mathrm{MnCl}_{2}$ were added to the growth medium. The protein was purified through nickel-agarose affinity chromatography (Qiagen) followed by gel filtration chromatography (Sephacryl S-300, GE Healthcare). The purified protein was concentrated to $15 \mathrm{mg} \mathrm{ml}^{-1}$ in a buffer containing $20 \mathrm{mM}$ Tris $(\mathrm{pH} 8.0), 150 \mathrm{mM}$ $\mathrm{NaCl}, 5 \%(\mathrm{v} / \mathrm{v})$ glycerol and $5 \mathrm{mM}$ dithiothreitol, flash-frozen in liquid nitrogen and stored at $-80^{\circ} \mathrm{C}$. The protein was confirmed to be fully biotinylated by a streptavidin gel-shift assay. The $\mathrm{N}$-terminal hexa-histidine tag was not removed for crystallization.

Protein crystallization. Crystals were grown by the sitting-drop vapour diffusion method at $20^{\circ} \mathrm{C}$. For MfPC with B-domain deletion, the protein was incubated with $2.5 \mathrm{mM}$ pyruvate for $30 \mathrm{~min}$ at $4{ }^{\circ} \mathrm{C}$ before crystallization set-up. The reservoir solution contained 19\% (w/v) PEG3350, 2\% tacsimate (pH 6.0) (Hampton) and 3\% (v/v) ethanol. The crystals appeared within 1 week and grew to full size after an additional week. The crystals were cryo-protected in the reservoir solution supplemented with $10 \%(\mathrm{v} / \mathrm{v})$ ethylene glycol and $5 \%(\mathrm{w} / \mathrm{v})$ sucrose and were flash-frozen in liquid nitrogen for data collection at $100 \mathrm{~K}$.

For wild-type MfPC, the protein at $15 \mathrm{mg} \mathrm{m}^{-1}$ was incubated with $2.5 \mathrm{mM}$ ATP and $2.5 \mathrm{mM}$ pyruvate for $30 \mathrm{~min}$ at $4{ }^{\circ} \mathrm{C}$ before crystallization set-up. The reservoir solution contained $1.3 \mathrm{M}$ ammonium sulfate and $0.1 \mathrm{M}$ sodium citrate $(\mathrm{pH} 6.0)$. The crystals appeared after 1 day and grew to full size within a week. They were cryo-protected in the reservoir solution supplemented with $15 \%(\mathrm{v} / \mathrm{v})$ ethylene glycol and flash-frozen in liquid nitrogen for data collection at $100 \mathrm{~K}$

Data collection and structure determination. X-ray diffraction data were collected at the Advanced Photon Source beamline NE-CAT 24-ID-E using an ADSC Q315r detector and at the X25 beamline at the National Synchrotron Light Source at Brookhaven National Laboratory using a Pilatus $6 \mathrm{M}$ detector. The diffraction images were processed using HKL2000 (ref. 37).

Crystals of the mutant MfPC belong to space group $R 32$ with unit cell parameters of $a=b=285.8 \AA$ and $c=274.9 \AA$. With an $\alpha_{2} \beta_{4}$ oligomer in the asymmetric unit, the $V_{\mathrm{m}}$ is $2.9 \AA^{3} \mathrm{Da}^{-1}$ and the solvent content is $58 \%$. The structure was solved by the molecular replacement method with the programme Phaser ${ }^{38}$, using the BC, CT and BCCP domains of the S. aureus PC structure ${ }^{9}$ as the search models. Manual rebuilding was carried out with $\operatorname{Coot}^{39}$ and refinement with the programme Refmac ${ }^{40}$.

Crystals of wild-type MfPC belong to space group $P 3_{1} 21$ with unit cell parameters of $a=b=160.8 \AA$ and $c=227.7 \AA$. A molecular replacement solution for this structure was found with the programme Phaser using the individual BC and CT domains of the refined mutant MfPC structure as the search models. There is one $\alpha$ subunit and two $\beta$ subunits in the asymmetric unit, and the full $\alpha_{2} \beta_{4}$ complex can be generated by a crystallographic two-fold axis. On the basis of this molecular replacement solution, the solvent content of the crystal is $73 \%$ and the Matthews coefficient is $4.6 \AA^{3} \mathrm{Da}^{-1}$. After one round of refinement, electron density was observed indicating the positions of the $\mathrm{B}$ domain of $\mathrm{BC}$ and the BT-like domain. There was also density indicating a BCCP domain in the active site of the distal CT domain, which itself adopts a conformation that is consistent with an interaction with BCCP. In addition, the MfPC molecules in the crystal do not have direct contacts without this BCCP domain, further supporting its placement in the model. After inclusion of these additional domains, we observed a decrease in the $R$ values and clear density for these domains on refinement.

Analytical ultracentrifugation. Analytical ultracentrifugation was performed on an XL-A analytical ultracentrifuge (Beckman Coulter) using an An-50 Ti rotor. The sedimentation velocity experiments were carried out using a double-sector epon charcoal-filled centrepiece at $20^{\circ} \mathrm{C}$ with a rotor speed of 42,000 r.p.m. Protein solutions of $0.5 \mathrm{mg} \mathrm{m}^{-1}(330 \mu \mathrm{l})$ and reference $(370 \mu \mathrm{l})$ solutions, both containing $20 \mathrm{mM}$ Tris $(\mathrm{pH} 8.0)$ and $150 \mathrm{mM} \mathrm{NaCl}$, were loaded into the centrepiece. The absorbance at $280 \mathrm{~nm}$ was monitored in a continuous mode with a time interval of $360 \mathrm{~s}$ and a step size of $0.003 \mathrm{~cm}$. Multiple scans at different time intervals were then fitted to a continuous $\mathrm{c}(\mathrm{s})$ distribution model using the SEDFIT programme ${ }^{41}$

Mutagenesis and kinetic studies. Mutants were made using the QuikChange kit (Stratagene) and confirmed by sequencing. They were expressed and purified using the same protocol as described for the wild-type enzyme. The catalytic activity was determined based on a published protocol ${ }^{42}$, which couples oxaloacetate production to the oxidation of NADH by malate dehydrogenase, followed spectrophotometrically by the decrease in absorbance at $340 \mathrm{~nm}$. The activity was measured at room temperature in a reaction mixture containing $20 \mathrm{mM}$ Tris (pH 7.5), $200 \mathrm{mM} \mathrm{NaCl}, 5 \mathrm{mM} \mathrm{MgCl}_{2}, 50 \mathrm{mM}$ sodium bicarbonate, $50 \mathrm{mM}$ ammonium sulfate, 5 units of malate dehydrogenase (Sigma), 2 mM ATP, $108 \mathrm{nM}$ MfPC (based on the $\alpha$ subunit) and varying concentrations of pyruvate. The Michaelis-Menten curve was fitted using the programme Origin.

Construction of PC mutant and complementation strains. PC mutant and complementation strains were made for the two-gene operon encoding PC (PA14_71720-PA14_71740) in P. aeruginosa PA14 as follows. Relevant genomic sequences were amplified, with point mutations introduced where noted in Supplementary Table 1, and recombined into the allelic replacement vector pMQ30 through gap repair cloning in the Saccharomyces cerevisiae strain InvScl. The deletion construct contained two fused $\sim 1-\mathrm{kb}$ sequences representing regions upstream and downstream of the PA14_71720-PA14_71740 operon. Point-mutant and complementation constructs contained the full operon sequence, with mutations where appropriate, plus these two flanking regions. Each plasmid was transformed into $E$. coli strain $\mathrm{DH} 5 \alpha$, verified by sequencing, and put into $P$. aeruginosa using biparental conjugation. PA14 single recombinants were selected on LB plates containing $100 \mu \mathrm{g} \mathrm{ml}^{-1}$ gentamicin. Double recombinants (with the final genotype of interest) were selected on agar plates containing $10 \%(\mathrm{w} / \mathrm{v})$ sucrose, and their genotypes were confirmed by PCR. For point mutant strains, genotypes were confirmed by sequencing. 
P. aeruginosa PA14 growth conditions. For genetic manipulation and pre-culturing, PA14 was routinely grown in $\mathrm{LB}$ (unless otherwise noted) at $37^{\circ} \mathrm{C}$ with shaking at 250 r.p.m. For growth curve analysis, overnight pre-cultures of PA14 strains were diluted 100 -fold in either $1 \%$ tryptone or a defined medium (50 mM MOPS (4-morpholinepropanesulfonic acid), $43 \mathrm{mM} \mathrm{NaCl}, 93.5 \mathrm{mM}$ $\mathrm{NH}_{4} \mathrm{Cl}, 2.2 \mathrm{mM} \mathrm{KH}_{2} \mathrm{PO}_{4}, 1 \mathrm{mM} \mathrm{MgSO}$ and $1 \mu \mathrm{gml}^{-1} \mathrm{FeSO}_{4}$ ) amended with $20 \mathrm{mM}$ sodium succinate, sodium pyruvate or glucose as the carbon source and grown to the following approximate $\mathrm{OD}_{500 \mathrm{~nm}}$ values: 0.6 for succinate; 0.1 for pyruvate; and 0.1 for glucose. These cultures were diluted to $\mathrm{OD}_{500 \mathrm{~nm}} \approx 0.01$ and dispensed into 96 -well plates, then incubated at $37^{\circ} \mathrm{C}$ with continuous shaking on the medium setting in a Biotek Synergy 4 plate reader.

P. aeruginosa PA14 colony morphology assay. For the standard assay, PA14 was grown overnight in $\mathrm{LB}$ and diluted to $\mathrm{OD}_{500 \mathrm{~nm}}=0.5$. A volume of $10 \mu \mathrm{l}$ of these normalized cell suspensions were spotted on colony morphology assay medium ( $1 \%$ tryptone, $1 \%$ agar, $40 \mu \mathrm{g} \mathrm{ml}^{-1}$ Congo red and $20 \mu \mathrm{g} \mathrm{ml}^{-1}$ Coomassie blue; $60 \mathrm{ml}$ in each $9 \mathrm{~cm} \times 9 \mathrm{~cm}$ square plate) and incubated in a humidified chamber at $25^{\circ} \mathrm{C}$ for up to 5 days. When defined media were used for the colony morphology assay, PA14 pre-cultures were grown overnight in MOPS liquid medium (50 mM MOPS, $43 \mathrm{mM} \mathrm{NaCl}, 93.5 \mathrm{mM} \mathrm{NH}_{4} \mathrm{Cl}, 2.2 \mathrm{mM} \mathrm{KH}_{2} \mathrm{PO}_{4}, 1 \mathrm{mM} \mathrm{MgSO}$ and $1 \mu \mathrm{g} \mathrm{ml}^{-1}$ $\mathrm{FeSO}_{4}$ with $20 \mathrm{mM}$ succinate). Pre-cultures were centrifuged for $1 \mathrm{~min}$ at $12,396 \times \mathrm{g}$ and resuspended in defined medium without a carbon source to $\mathrm{OD}_{500} \mathrm{~nm}=0.25$. A volume of $10 \mu \mathrm{l}$ of washed and resuspended cells were spotted onto solidified defined medium (25 mM HEPES (4-(2-hydroxyethyl)-1-piperazineethanesulfonic acid), $7.6 \mathrm{mM}\left(\mathrm{NH}_{4}\right)_{2} \mathrm{SO}_{4}, 0.8 \mathrm{mM} \mathrm{MgSO} \mathrm{M}_{4} \cdot 7 \mathrm{H}_{2} \mathrm{O}, 10 \mathrm{mM}$ $\mathrm{K}_{2} \mathrm{HPO}_{4}, 20 \mathrm{mM}$ of carbon source (succinate, pyruvate or glucose), $40 \mu \mathrm{g} \mathrm{ml}^{-1}$ Congo red, $20 \mu \mathrm{g} \mathrm{ml}^{-1}$ Coomassie blue and $1 \%$ agar; $60 \mathrm{ml}$ in each $9 \mathrm{~cm} \times 9 \mathrm{~cm}$ square plate) and incubated in a humidified chamber at $25^{\circ} \mathrm{C}$ for up to 5 days. Images were taken with a CanoScan 5600F scanner (Canon).

P. aeruginosa PA14 Congo red binding assay. PA14 colonies were grown for 3 days $(76 \mathrm{~h})$ in the standard colony morphology assay, with the modification that dyes were omitted from the $1 \%$ tryptone, $1 \%$ agar medium. Each colony was scraped from the agar using a $1,000 \mu \mathrm{l}$ pipette tip and resuspended in $1.5 \mathrm{ml}$ phosphate-buffered saline (136 mM NaCl, $2.68 \mathrm{mM} \mathrm{KCl}, 10.1 \mathrm{mM} \mathrm{Na}_{2} \mathrm{HPO}_{4}$ and $1.76 \mathrm{mM} \mathrm{KH}_{2} \mathrm{PO}_{4}$, at pH 7.4) supplemented with $60 \mu \mathrm{g}$ of Congo red. Each colony suspension was briefly vortexed and shaken at 250 r.p.m. at $37^{\circ} \mathrm{C}$ for $1 \mathrm{~h}$ to allow the matrix to bind the Congo red dye. The colony biomass was then pelleted by centrifugation at $16,873 \times \mathrm{g}$ for $2 \mathrm{~min}$. A volume of $200 \mu \mathrm{l}$ of supernatant were dispensed into 96 -well plates and the absorbance at $490 \mathrm{~nm}$, representing unbound Congo red, was measured using a Biotek Synergy 4 plate reader. Bound Congo red was calculated by subtracting the absorbance of the unbound Congo red from that of the control solution containing $60 \mu \mathrm{g}$ Congo red per $1.5 \mathrm{ml}$ PBS.

P. aeruginosa PA14 pellicle assay. Overnight LB pre-cultures of PA14 strains were diluted 100 -fold in MOPS liquid medium $(50 \mathrm{mM}$ MOPS, $43 \mathrm{mM} \mathrm{NaCl}$, $93.5 \mathrm{mM} \mathrm{NH}_{4} \mathrm{Cl}, 2.2 \mathrm{mM} \mathrm{KH}_{2} \mathrm{PO}_{4}, 1 \mathrm{mM} \mathrm{MgSO}_{4}$ and $1 \mu \mathrm{g} \mathrm{ml}^{-1} \mathrm{FeSO}_{4}$ ) amended with $20 \mathrm{mM}$ sodium succinate as the carbon source and grown to $\mathrm{OD}_{500 \mathrm{~nm}} \approx 0.7$ A sample $(4.6 \mathrm{ml})$ of each subculture was centrifuged at $10,000 \times \mathrm{g}$ for $1 \mathrm{~min}$, and the pellet was resuspended in $23 \mathrm{ml}$ of the defined medium amended with $20 \mathrm{mM}$ sodium succinate, D-glucose or sodium pyruvate in a scintillation vial, with a starting $\mathrm{OD}_{500 \mathrm{~nm}}$ at 0.14 for pellicle growth. Scintillation vials were incubated without shaking or disturbance at $37^{\circ} \mathrm{C}$ for up to 4 days and photographed under side illumination using an iPhone $5 \mathrm{~S}$.

Data availability. Atomic coordinates and structure factors for the two reported structures of M. flagellatus PC have been deposited in the Protein Data Bank under the primary accession code $5 \mathrm{KS} 8$. The authors declare that all other relevant data supporting the findings of this study are available on request.

\section{References}

1. Jitrapakdee, S. et al. Structure, mechanism and regulation of pyruvate carboxylase. Biochem. J. 413, 369-387 (2008).

2. Tong, L. Structure and function of biotin-dependent carboxylases. Cell. Mol. Life Sci. 70, 863-891 (2013).

3. Sellers, K. et al. Pyruvate carboxylase is critical for non-small-cell lung cancer proliferation. J. Clin. Invest. 125, 687-698 (2015).

4. Phannasil, P. et al. Pyruvate carboxylase is up-regulated in breast cancer and essential to support growth and invasion of MDA-MB-231 cells. PLoS ONE 10, e0129848 (2015).

5. Schar, J. et al. Pyruvate carboxylase plays a crucial role in carbon metabolism of extra- and intracellularly replicating Listeria monocytogenes. J. Bacteriol. 192, 1774-1784 (2010).

6. Sureka, K. et al. The cyclic dinucleotide c-di-AMP is an allosteric regulator of metabolic enzyme function. Cell 158, 1389-1401 (2014).

7. Perry, R. J. et al. Hepatic acetyl CoA links adipose tissue inflammation to hepatic insulin resistance and type 2 diabetes. Cell 160, 745-758 (2015).
8. St Maurice, M. et al. Domain architecture of pyruvate carboxylase, a biotindependent multifunctional enzyme. Science 317, 1076-1079 (2007).

9. Xiang, S. \& Tong, L. Crystal structures of human and Staphylococcus aureus pyruvate carboxylase and molecular insights into the carboxyltransfer reaction. Nat. Struct. Mol. Biol. 15, 295-302 (2008).

10. Yu, L. P. C. et al. A symmetrical tetramer for S. aureus pyruvate carboxylase in complex with coenzyme A. Structure 17, 823-832 (2009).

11. Lai, H., Kraszewski, J. L., Purwantini, E. \& Mukhopadhyay, B. Identification of pyruvate carboxylase genes in Pseudomonas aeruginosa PAO1 and development of a $P$. aeruginosa-based overexpression system for $\mathrm{a} 4-$ and $\mathrm{a} 4 \mathrm{~b} 4$ type pyruvate carboxylases. Appl. Environ. Microbiol 72, 7785-7792 (2006).

12. Cohen, N. D., Duc, J. A., Beegen, H. \& Utter, M. F. Quaternary structure of pyruvate carboxylase from Pseudomonas citronellolis. J. Biol. Chem. 254, 9262-9269 (1979).

13. Adina-Zada, A., Zeczycki, T. N. \& Attwood, P. V. Regulation of the structure and activity of pyruvate carboxylase by acetyl CoA. Arch. Biochem. Biophys. 519, 118-130 (2012).

14. Phibbs, Jr P. V., Feary, T. W. \& Blevins, W. T. Pyruvate carboxylase deficiency in pleiotropic carbohydrate-negative mutant strains of Pseudomonas aeruginosa. J. Bacteriol. 118, 999-1009 (1974).

15. Kukavica-Ibrulj, I. et al. Functional genomics of PycR, a LysR family transcriptional regulator essential for maintenance of Pseudomonas aeruginosa in the rat lung. Microbiology 154, 2106-2118 (2008).

16. Takeuchi, K. et al. Small RNA-dependent expression of secondary metabolism is controlled by Krebs cycle function in Pseudomonas fluorescens. J. Biol. Chem. 284, 34976-34985 (2009).

17. Scrutton, M. C. \& Taylor, B. L. Isolation and characterization of pyruvate carboxylase from Azotobacter vinelandii OP. Arch. Biochem. Biophys. 164, 641-654 (1974).

18. Segura, D. \& Espin, G. Inactivation of pycA, encoding pyruvate carboxylase activity, increases poly-b-hydroxybutyrate accumulation in Azotobacter vinelandii on solid medium. Appl. Microbiol. Biotechnol. 65, 414-418 (2004).

19. Kondo, S. et al. Structure of the biotin carboxylase subunit of pyruvate carboxylase from Aquifex aeolicus at $2.2 \AA$ resolution. Acta Crystallogr. D Biol. Crystallogr. D60, 486-492 (2004).

20. Huang, C. S. et al. Crystal structure of the a6b6 holoenzyme of propionylcoenzyme A carboxylase. Nature 466, 1001-1005 (2010).

21. Huang, C. S., Ge, P., Zhou, Z. H. \& Tong, L. An unanticipated architecture of the $750-\mathrm{kDa}$ a6b6 holoezyme of 3-methylcrotonyl-CoA carboxylase. Nature 481, 219-223 (2012).

22. Wei, J. \& Tong, L. Crystal structure of the $500-\mathrm{kDa}$ yeast acetyl-CoA carboxylase holoenzyme dimer. Nature 526, 723-727 (2015).

23. Chistoserdova, L. et al. Genome of Methylobacillus flagellatus, molecular basis for obligate methylotrophy, and polyphyletic origin of methylotrophy. J. Bacteriol. 189, 4020-4027 (2007).

24. Goldschmidt, L., Cooper, D. R., Derewenda, Z. S. \& Eisenberg, D. Toward rational protein crystallization: a web server for the design of crystallizable protein variants. Protein Sci. 16, 1569-1576 (2007).

25. Yu, L. P. C., Chou, C.-Y., Choi, P. H. \& Tong, L. Characterizing the importance of the biotin carboxylase domain dimer for Staphylococcus aureus pyruvate carboxylase catalysis. Biochemistry 52, 488-496 (2013).

26. Milrad de Forchetti, S. R. \& Cazzulo, J. J. Some properties of the pyruvate carboxylase from Pseudomonas fluorescens. J. Gen. Microbiol. 93, 75-81 (1976).

27. Barden, R. E. et al. Structural properties of pyruvate carboxylases from chicken liver and other sources. Proc. Natl Acad. Sci. USA 72, 4308-4312 (1975).

28. Waldrop, G. L., Rayment, I. \& Holden, H. M. Three-dimensional structure of the biotin carboxylase subunit of acetyl-CoA carboxylase. Biochemistry 33, 10249-10256 (1994).

29. Shen, Y., Chou, C.-Y., Chang, G.-G. \& Tong, L. Is dimerization required for the catalytic activity of bacterial biotin carboxylase? Mol. Cell 22, 807-818 (2006).

30. Friedman, L. \& Kolter, R. Genes involved in matrix formation in Pseudomonas aeruginosa PA14 biofilms. Mol. Microbiol. 51, 675-690 (2004).

31. Madsen, J. S. et al. Facultative control of matrix production optimizes competitive fitness in Pseudomonas aeruginosa PA14 biofilm models. Appl. Environ. Microbiol. 81, 8414-8426 (2015).

32. Dietrich, L. E. P. et al. Bacterial community morphogenesis is intimately linked to the intracellular redox state. J. Bacteriol. 195, 1371-1380 (2013).

33. Jennings, L. K. et al. Pel is a cationic exopolysaccharide that cross-links extracellular DNA in the Pseudomonas aeruginosa biofilm matrix. Proc. Natl Acad. Sci. USA 112, 11353-11358 (2015).

34. Cronan, Jr J. E. \& Waldrop, G. L. Multi-subunit acetyl-CoA carboxylases. Prog. Lipid Res. 41, 407-435 (2002).

35. Mukhopadhyay, B., Stoddard, S. F. \& Wolfe, R. S. Purification, regulation, and molecular and biochemical characterization of pyruvate carboxylase from Methanobacterium thermoautotrophicum strain deltaH. J. Biol. Chem. 273, 5155-5166 (1998). 
36. Mukhopadhyay, B., Patel, V. J. \& Wolfe, R. S. A stable archaeal pyruvate carboxylase from the hyperthermophile Methanococcus jannaschii. Arch. Microbiol. 174, 406-414 (2000).

37. Otwinowski, Z. \& Minor, W. Processing of X-ray diffraction data collected in oscillation mode. Method Enzymol. 276, 307-326 (1997).

38. McCoy, A. J. et al. Phaser crystallographic software. J. Appl. Cryst. 40, 658-674 (2007).

39. Emsley, P. \& Cowtan, K. D. Coot: model-building tools for molecular graphics. Acta Crystallogr. D Biol. Crystallogr. D60, 2126-2132 (2004).

40. Murshudov, G. N., Vagin, A. A. \& Dodson, E. J. Refinement of macromolecular structures by the maximum-likelihood method. Acta Crystallogr. D Biol. Crystallogr. D53, 240-255 (1997).

41. Schuck, P. Size-distribution analysis of macromolecules by sedimentation velocity ultracentrifugation and Lamm equation modeling. Biophys. J. 78, 1606-1619 (2000).

42. Modak, H. V. \& Kelly, D. J. Acetyl-CoA-dependent pyruvate carboxylase from the photosynthetic bacterium Rhodobacter capsulatus: rapid and efficient purification using dye-ligand affinity chromatography. Microbiology 141, 2619-2628 (1995).

\section{Acknowledgements}

We thank A. Price-Whelan for discussions; R. Jackimowicz, N. Whalen, A. Heroux and H. Robinson for access to the X25 beamline of National Synchrotron Light Source; and S. Banerjee, K. Perry, R. Rajashankar, J. Schuermann and N. Sukumar for access to NE-CAT 24-ID-E beamline at the Advanced Photon Source. This research is supported by grants from the NIH (R01DK067238 and S10OD012018 to L.T. and R01AI103369 to L.E.P.D.) and by a grant from the Ministry of Science and Technology, Taiwan (MOST 104-2320-B-010-034) to C.-Y.C. P.H.C. is also supported by an NIH Medical Scientist Training Program (GM007367).

\section{Author contributions}

Designed and performed crystallographic experiments: P.H.C. and L.T.; designed and performed P. aeruginosa studies: J.J., Y.-C.L. and L.E.P.D.; designed and performed AUC experiments: M.-H.L. and C.-Y.C.; wrote the paper: P.H.C., C.-Y.C., L.E.P.D. and L.T. All authors commented on the manuscript.

\section{Additional information}

Supplementary Information accompanies this paper at http://www.nature.com/ naturecommunications

Competing financial interests: The authors declare no competing financial interests.

Reprints and permission information is available online at http://npg.nature.com/ reprintsandpermissions/

How to cite this article: Choi, P. H. et al. A distinct holoenzyme organization for two-subunit pyruvate carboxylase. Nat. Commun. 7:12713 doi: 10.1038/ncomms12713 (2016).

(c) (i) This work is licensed under a Creative Commons Attribution 4.0 International License. The images or other third party material in this article are included in the article's Creative Commons license, unless indicated otherwise in the credit line; if the material is not included under the Creative Commons license, users will need to obtain permission from the license holder to reproduce the material. To view a copy of this license, visit http://creativecommons.org/licenses/by/4.0/

C) The Author(s) 2016 